Pacific

Journal of

Mathematics

\title{
ITERATED LOOP ALGEBRAS
}

Bruce Allison, Stephen Berman and Arturo Pianzola 


\title{
ITERATED LOOP ALGEBRAS
}

\author{
Bruce Allison, Stephen Berman and Arturo Pianzola
}

\begin{abstract}
Iterated loop algebras are by definition obtained by repeatedly applying the loop construction, familiar from the theory of affine Kac-Moody Lie algebras, to a given base algebra. Our interest in this iterated construction is motivated by its use in the realization of extended affine Lie algebras, but the construction also appears naturally in the study of other classes of algebras. This paper consists of a detailed study of the basic properties of iterated loop algebras.
\end{abstract}

\section{Introduction}

Over the past 35 years affine Kac-Moody Lie algebras have been at the centre of a considerable amount of beautiful mathematics and theoretical physics. As of late, and perhaps influenced by some of the newest theories in physics, the need seems to have arisen for some "higher nullity" generalizations of affine KacMoody Lie algebras. It is still too early to decide what the correct final choice for these algebras will be, but it is fair to say notwithstanding, that Lie algebras graded by root systems and extended affine Lie algebras (EALAs) will play a prominent role in the process [Berman and Moody 1992; Benkart and Zelmanov 1996; Allison et al. 1997a; Saito and Yoshii 2000].

Recall that given a $\mathbb{Z}_{m}$-grading $\Sigma=\left\{\mathscr{A}_{\bar{l}}\right\}_{\bar{i} \in \mathbb{Z}_{m}}$ of an algebra $\mathscr{A}$ over a field $k$, the loop algebra of $\Sigma$ based on $\mathscr{A}$ is the subalgebra

$$
L(\mathscr{A}, \Sigma):=\bigoplus_{i \in \mathbb{Z}} \mathscr{A}_{\bar{l}} \otimes_{k} z^{i}
$$

of $\mathscr{A} \otimes_{k} k\left[z, z^{-1}\right]$. Using this beautiful construction, V. Kac showed that (the derived algebra modulo its centre of) any complex affine Kac-Moody Lie algebras can be obtained as a loop algebra of a finite dimensional simple Lie algebra [Kac 1969]. The loop construction makes it clear, among other things, that the affine algebras

MSC2000: primary 17B65; secondary 17B67, 16S99, 17C99, 17D05, 17A01.

Keywords: loop algebra, Lie algebra, associative algebra, Jordan algebra.

The authors gratefully acknowledge the support of the Natural Sciences and Engineering Research Council of Canada. 
are objects of nullity one in a sense that can be made precise. Indeed, in EALA theory, where the concept of nullity is well-defined, one finds that finite dimensional simple algebras are precisely the (tame) EALAs of nullity zero whereas affine algebras are precisely the (tame) EALAs of nullity one [Allison et al. 1997b].

It thus seems almost inevitable to ask whether, starting from an affine KacMoody Lie algebra and applying the loop construction, one obtains an extended affine Lie algebra of nullity 2. This and related questions have been investigated in some detail in [Wakimoto 1985; Pollmann 1994; Allison et al. 2002; Allison et al. 2004; van de Leur 2001]. In our work on this topic, as well as in [van de Leur 2001], it became clear that some advantages are to be had by thinking of loop algebras based on an affine algebra as being obtained from a finite dimensional Lie algebra by applying the loop construction twice (the advantages stemming from the fact that in this case the "bottom" algebra, namely the finite dimensional one, is much simpler than the affine algebra). As the reader will have surmised by now, the study of these "iterated loop algebras" took on a life of its own and became the subject of the present paper.

In general, if $\mathscr{A}$ is an (arbitrary) algebra over $k$, an $n$-step iterated loop algebra based on $\mathscr{A}$ is an algebra that can be obtained starting from $\mathscr{A}$ by a sequence of $n$ loop constructions, each based on the algebra obtained at the previous step (see Definition 5.1). Far from being a mere generalization of the loop construction, iterated loop algebras seem to yield interesting mathematical objects in a natural way. Even when the resulting objects are known, the new point of view can be illuminating. As an example, we see in Example 9.8 that algebras representing elements of the Brauer group of the ring $k\left[t_{1}^{ \pm 1}, t_{2}^{ \pm 2}\right]$ are obtained as 2-step iterated loop algebras of $M_{n}(k)$. This information is not apparent if one thinks in terms of single loop algebras of $M_{\ell}\left(k\left[t_{1}^{ \pm 1}\right]\right)$.

This paper contains a detailed study of the basic properties of iterated loop algebras. We begin in Section 2 by recording some simple properties of the centroid of an algebra. In the rest of Section 2 and in Section 3 we define and give the basic properties of a very important class of algebras which for lack of a better name we have simply referred to as pfgc algebras (nonzero, perfect, and finitely generated as modules over their centroids). The property of being a prime pfgc algebra arises naturally in the study of iterated loop algebras since this property is carried over to a loop algebra (and hence to an iterated loop algebra) from its base. In contrast the property of finite dimensional central simplicity certainly does not carry over in the same way. After this discussion of pfgc algebras we establish in Section 4 some basic properties of (one step) loop algebras.

The main results of the paper appear in Sections 5, 6, 7 and 8 . These all deal with properties of an $n$-step iterated loop algebra $\mathscr{L}$ based on a pfgc algebra $\mathscr{A}$. First Theorem 5.5 establishes a long list of properties that carry over from $\mathscr{A}$ to $\mathscr{L}$. 
In particular, it is shown (as mentioned above) that if $\mathscr{A}$ is a prime pfgc algebra then so is $\mathscr{L}$. Next Theorem 6.2 shows that the centroid $C(\mathscr{L})$ of $\mathscr{L}$ is itself an $n$-step iterated loop algebra of the centroid of $\mathscr{A}$. The same theorem describes a method of calculating $C(\mathscr{L})$ explicitly. Then Theorem 7.1 shows that $\mathscr{L}$ can be "untwisted" by a base ring extension of $C(\mathscr{L})$ that is free of finite rank. That is, the algebra $\mathscr{L}$ (after such a base ring extension) becomes isomorphic to the iterated loop algebra obtained using only the trivial gradings at each stage. Finally, Section 8 deals with the concept of type of an algebra (which is motivated by the concept of type in terms of root systems which exists in Lie theory). The main result, Theorem 8.16, states that type cannot change under the loop construction.

Each of the main results in Sections 6, 7 and 8 has several corollaries that are discussed in the respective sections. To give one important example, we show in Section 8 that if $\mathscr{L}$ is an $n$-step iterated loop algebra based on a finite dimensional split simple Lie algebra $\mathscr{A}$ over a field of characteristic 0 then both $\mathscr{A}$ and $n$ are isomorphism invariants of $\mathscr{L}$ (see Corollary 8.19). This result will play a crucial role in our forthcoming work on the classification of the centreless cores of EALAs of nullity 2 [Allison et al. $\geq 2006$ ].

In the last section, Section 9, we look closely at 2-step iterated loop algebras. If the base algebra is finite dimensional and central simple, these 2-step iterated loop algebras come in two kinds, depending on the structure of their centroids. We illustrate this fact along with many of the concepts discussed in the paper by describing two examples dealing respectively with Lie algebras and associative algebras.

\section{Centroids and pfgc algebras}

We record here some basic facts about centroids, and we define a class of algebras, which we call pfgc algebras, that will play an important role in the study of loop algebras. A good basic reference on the centroid is [Jacobson 1962, Ch. X, § 1].

Terminology and notation. A ring will mean a unital commutative associative ring. Homomorphisms, subrings and modules for rings will always be assumed to be unital.

A base change will mean a homomorphism $v: B \rightarrow B^{\prime}$ of rings. This base change is said to be free (respectively flat, faithfully flat) if $B^{\prime}$ is a free (respectively flat, faithfully flat) $B$-module. Note that if $v: B \rightarrow B^{\prime}$ is free and $B^{\prime} \neq 0$, then $v$ is faithfully flat and hence flat [Bourbaki 1972, § I.3.1, Example 2]. An injective base change $v: B \rightarrow B^{\prime}$ will be called an extension of rings, in which case we often identify $B$ as a subring of $B^{\prime}$ and denote the extension by $B^{\prime} / B$.

If $B$ is a ring, an algebra over $B$ will mean a $B$-module $A$ together with a $B$ bilinear product (which is not necessarily associative, commutative or unital). If 
$\mathscr{A}$ and $\mathscr{A}^{\prime}$ are $B$-algebras, we use the notation

$$
\mathscr{A} \simeq_{B} \mathscr{A}^{\prime}
$$

to mean that $\mathscr{A}$ and $\mathscr{A}^{\prime}$ are isomorphic as $B$-algebras. If $\mathscr{A}$ is an algebra over $B$ and $v: B \rightarrow B^{\prime}$ is a base change, we will denote by $\mathscr{A} \otimes_{B} B^{\prime}$ the (unique) $B^{\prime}$-algebra which is obtained from $\mathscr{A}$ by base change [Bourbaki 1974, Ch. III, $\S 1.5$ ].

For the rest of the section we assume that $B$ is a ring, and that $\mathcal{A}$ is a B-algebra. Note that $\mathscr{A}$ can also be regarded as $\mathbb{Z}$-algebra under the natural action of $\mathbb{Z}$ on $\mathscr{A}$.

We now recall the definition of the centroid of $\mathscr{A}$ [Jacobson 1962, Ch. X, §1].

Definition 2.1. (i) For $a \in \mathscr{A}$ consider the two maps from $\mathscr{A}$ to $\mathscr{A}$

$$
a_{L}: x \mapsto a x \quad \text { and } \quad a_{R}: x \mapsto x a .
$$

The multiplication algebra of $\mathscr{A}$ [Jacobson 1962, Ch. X, $\S 1$ ] is defined to be the $B$ subalgebra $\operatorname{Mult}_{B}(\mathscr{A})$ of $\operatorname{End}_{B}(\mathscr{A})$ generated by $\{1\} \cup\left\{a_{L} \mid a \in \mathscr{A}\right\} \cup\left\{a_{R} \mid a \in \mathscr{A}\right\}$.

(ii) The set $C_{B}(\mathscr{A})$ of elements of $\operatorname{End}_{B}(\mathscr{A})$ that commute with the action of $\operatorname{Mult}_{B}(\mathscr{A})$ is called the centroid of $\mathscr{A}$. Equivalently

$$
C_{B}(\mathscr{A}):=\left\{\chi \in \operatorname{End}_{B}(\mathscr{A}): \chi(x y)=\chi(x) y=x \chi(y) \text { for all } x, y \in \mathscr{A}\right\} .
$$

(The notation $\operatorname{Cent}_{B}(\mathscr{A})$ has been used for the centroid in some articles, for example in [Allison et al. 2004]. We are using the abbreviated notation $C_{B}(\mathscr{A})$ since it will arise frequently.) Clearly $C_{B}(\mathscr{A})$ is a $B$-subalgebra of $\operatorname{End}_{B}(\mathscr{A})$, and therefore $\mathscr{A}$ can be viewed in a natural way as a left $C_{B}(\mathscr{A})$-module by defining $\chi \cdot x=\chi(x)$.

(iii) For $b \in B$ we define $\lambda_{\mathscr{A}}(b) \in \operatorname{End}_{B}(\mathscr{A})$ by

$$
\left(\lambda_{\mathscr{A}}(b)\right)(x)=b \cdot x .
$$

Clearly $\lambda_{\mathscr{A}}(b) \in C_{B}(\mathscr{A})$ since $\mathscr{A}$ is a $B$-algebra. Then the map $\lambda_{\mathscr{A}}: B \rightarrow C_{B}(\mathscr{A})$ is a ring homomorphism, and $C_{B}(\mathscr{A})$ is a unital associative $B$-algebra via this map. Furthermore, if $\mathscr{A}$ is a faithful $B$-module then $B$ can be identified with a subring of (the centre of) the centroid $C_{B}(\mathscr{A})$.

(iv) The $B$-algebra $\mathscr{A}$ is said to be central (or central over $B$ ) if $\lambda_{\mathscr{A}}: B \rightarrow C_{B}(\mathscr{A})$ is an isomorphism.

(v) The centre of $\mathscr{A}$ is defined to be the set $Z(\mathscr{A})$ of elements in $\mathscr{A}$ that commute and associate with all elements of $\mathscr{A}$. Then $Z(\mathscr{A})$ is a $B$-subalgebra of $\mathscr{A}$. If $\mathscr{A}$ is unital, the map which sends $z$ to left multiplication by $z$ is a $B$-algebra isomorphism of $Z(\mathscr{A})$ onto $C_{B}(\mathscr{A})$ [Erickson et al. 1975, § 1].

The following is clear: 
Lemma 2.2. Suppose that $\mathscr{A}$ and $\mathscr{A}^{\prime}$ are $B$-algebras and $\rho: \mathscr{A} \rightarrow \mathscr{A}^{\prime}$ is a $B$-algebra isomorphism. Then $\rho$ induces a $B$-algebra isomorphism $C_{B}(\rho): C_{B}(\mathscr{A}) \rightarrow C_{B}\left(\mathscr{A}^{\prime}\right)$ defined by $\chi \mapsto \rho \chi \rho^{-1}$.

The formation of the centroid does not commute with base change. Nonetheless these two processes do commute in two important cases that we now describe. If $B \rightarrow B^{\prime}$ is a homomorphism of rings, we define

$$
v=v_{\mathscr{A}, B, B^{\prime}}: C_{B}(\mathscr{A}) \otimes_{B} B^{\prime} \rightarrow C_{B^{\prime}}\left(\mathscr{A} \otimes_{B} B^{\prime}\right)
$$

to be the restriction of the canonical map $\operatorname{End}_{B}(\mathscr{A}) \otimes_{B} B^{\prime} \rightarrow \operatorname{End}_{B^{\prime}}\left(\mathscr{A} \otimes_{B} B^{\prime}\right)$. Then $v$ is a homomorphism, said to be canonical, of unital associative $B^{\prime}$-algebras.

Lemma 2.3. Suppose that $B \rightarrow B^{\prime}$ is a homomorphism of rings. Then the map $v_{\mathscr{A}, B, B^{\prime}}: C_{B}(\mathscr{A}) \otimes_{B} B^{\prime} \rightarrow C_{B^{\prime}}\left(\mathscr{A} \otimes_{B} B^{\prime}\right)$ is an isomorphism of $B^{\prime}$-algebras in the following cases:

(a) $A$ is finitely generated as a module over its multiplication algebra $\operatorname{Mult}_{B}(\mathscr{A})$ and $B^{\prime}$ is a free $B$-module.

(b) $B^{\prime}$ is a finitely generated projective $B$-module.

Proof. (a) Let $\left\{s_{i}\right\}_{i \in I}$ be a basis of the $B$-module $B^{\prime}$.

It is clear that $v$ is injective. Indeed if $\sum \chi_{i} \otimes s_{i}$ is in the kernel of $v$ then $\sum \chi_{i}(x) \otimes s_{i}=0$ for all $x$ in $\mathscr{A}$ and so $\chi_{i}=0$ for all $i$ in $I$.

To see that $v$ is onto, let $\chi \in C_{B^{\prime}}\left(\mathscr{A} \otimes_{B} B^{\prime}\right)$. Then for $x \in \mathscr{A}$ we can write $\chi\left(x \otimes_{B} 1_{B^{\prime}}\right)$ uniquely as

$$
\chi\left(x \otimes 1_{B^{\prime}}\right)=\sum \chi_{i}(x) \otimes s_{i},
$$

where $\chi_{i}(x) \in \mathscr{A}$ and only finitely many of these are nonzero. It is easy to see that for all $i \in I$ the map $\chi_{i}: \mathscr{A} \rightarrow \mathscr{A}$ given by $\chi_{i}: x \mapsto \chi_{i}(x)$ is an element of $C_{B}(\mathscr{A})$. Thus to see that $\chi$ is an image under $v$ it suffices to show that only finitely many of the maps $\chi_{i}$ are nonzero. For this let $\left\{x_{1}, \ldots, x_{n}\right\}$ be a set of generators of $\mathscr{A}$ as a $\operatorname{Mult}_{B}(\mathscr{A})$-module. Then whenever $\chi_{i}$ vanishes on all $x_{j}$ 's we have

$$
\chi_{i}(\mathscr{A})=\chi_{i}\left(\sum_{j=1}^{n} \operatorname{Mult}_{B}(\mathscr{A}) \cdot x_{j}\right)=\sum_{j=1}^{n} \operatorname{Mult}_{B}(\mathscr{A}) \cdot \chi_{i}\left(x_{j}\right)=0 .
$$

(b) Consider the unique $B$-module homomorphism

$$
\varphi_{B, \mathscr{A}}: \operatorname{End}_{B}(\mathscr{A}) \rightarrow \operatorname{Hom}_{B}\left(\mathscr{A} \otimes_{B} \mathscr{A}, \mathscr{A} \oplus \mathscr{A}\right)
$$

satisfying

$$
\varphi_{B, \mathscr{A}}(f)\left(a_{1} \otimes_{B} a_{2}\right)=\left(f\left(a_{1} a_{2}\right)-f\left(a_{1}\right) a_{2}, f\left(a_{1} a_{2}\right)-a_{1} f\left(a_{2}\right)\right) .
$$


By definition

$$
\operatorname{ker}\left(\varphi_{B, \mathscr{A}}\right)=C_{B}(\mathscr{A})
$$

Also, by standard properties of projective modules we obtain the diagram

$0 \rightarrow C_{B}(\mathscr{A}) \otimes_{B} B^{\prime} \rightarrow \operatorname{End}_{B}(\mathscr{A}) \otimes_{B} B^{\prime} \rightarrow \operatorname{Hom}_{B}\left(\mathscr{A} \otimes_{B} \mathscr{A}, \mathscr{A} \oplus \mathscr{A}\right) \otimes_{B} B^{\prime}$

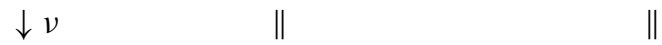

$0 \rightarrow C_{B^{\prime}}\left(\mathscr{A} \otimes_{B} B^{\prime}\right) \rightarrow \operatorname{End}_{B^{\prime}}\left(\mathscr{A} \otimes_{B} B^{\prime}\right) \rightarrow \operatorname{Hom}_{B^{\prime}}\left(\left(\mathscr{A} \otimes_{B} B^{\prime}\right) \otimes_{B^{\prime}}\left(\mathscr{A} \otimes_{B} B^{\prime}\right), \mathscr{A} \otimes_{B} B^{\prime} \oplus \mathscr{A} \otimes_{B} B^{\prime}\right)$

where the horizontal rows are exact. Indeed the exactness of the top row is by flatness of the $B$-module $B^{\prime}$ (every projective is flat). The two vertical isomorphisms come from $B^{\prime}$ being a finitely generated $B$-module which is projective [Bourbaki 1974, Ch. II, § 5.3, Prop. 7]. It follows that $v$ is an isomorphism.

The following important fact is proved in [Jacobson 1962, Ch. X, § 1, Theorem 3]:

Lemma 2.4. Suppose that $B$ is a field and $\mathscr{A}$ is finite dimensional and central simple over $B$. If $B^{\prime} / B$ is a field extension, then $\mathscr{A} \otimes_{B} B^{\prime}$ is finite dimensional and central simple over $B^{\prime}$.

Next we consider gradings on $C_{B}(A)$ that are induced by gradings on $\mathscr{A}$. For this suppose that $\mathscr{A}$ is $Q$-graded algebra over $B$ where $Q$ is a finite abelian group. Thus

$$
\mathscr{A}=\bigoplus_{\alpha \in Q} \mathscr{A}_{\alpha}
$$

for some $B$-submodules $\mathscr{A}_{\alpha}$ and $\mathscr{A}_{\alpha} \mathscr{A}_{\beta} \subset \mathscr{A}_{\alpha+\beta}$. Then, since $Q$ is finite,

$$
\operatorname{End}_{B}(\mathscr{A})=\bigoplus_{\lambda \in Q} \operatorname{End}_{B}(\mathscr{A})_{\lambda}
$$

is also a $Q$-graded $B$-algebra, where

$$
\operatorname{End}_{B}(\mathscr{A})_{\lambda}=\left\{\theta \in \operatorname{End}_{B}(\mathscr{A}) \mid \theta\left(\mathscr{A}_{\alpha}\right) \subset \mathscr{A}_{\lambda+\alpha} \text { for all } \alpha \in Q\right\} .
$$

It is easy to check that $C_{B}(\mathscr{A})$ is a $Q$-graded $B$-subalgebra of $\operatorname{End}_{B}(\mathscr{A})$, and so we have:

Lemma 2.5. Suppose that $\mathscr{A}$ is $Q$-graded algebra over $B$, where $Q$ is a finite abelian group. Then

$$
C_{B}(\mathscr{A})=\bigoplus_{\alpha \in Q} C_{B}(\mathscr{A})_{\lambda}
$$

is a $Q$-graded algebra over $B$, where $C_{B}(\mathscr{A})_{\lambda}=C_{B}(\mathscr{A}) \cap \operatorname{End}_{B}(\mathscr{A})_{\lambda}$ for all $\lambda \in Q$. 
Definition 2.6. If $\mathscr{I}$ and $\mathscr{F}$ are ideals of the $B$-algebra $\mathscr{A}$ we define

$$
\mathscr{I} \mathscr{F}=\left\{\sum x_{i} y_{i}: x_{i} \in \mathscr{I}, y_{i} \in \mathscr{F}\right\}
$$

(finite sums of course). Note that in general $\mathscr{\Phi}$ is not an ideal of $\mathscr{A}$. We say that $\mathscr{A}$ is perfect if $\mathscr{A} \mathscr{A}=\mathscr{A}$.

Remark 2.7. It is clear that $\mathscr{A}$ is perfect as a $B$-algebra if and only if $\mathscr{A}$ is perfect as a $\mathbb{Z}$-algebra.

Lemma 2.8. Assume $\mathscr{A}$ is perfect. Then

(i) $C_{B}(\mathscr{A})$ is commutative.

(ii) $C_{B}(\mathscr{A})=C_{\mathbb{Z}}(\mathscr{A})$.

Proof. (i) See [Jacobson 1962, Ch. X, § 1, Lemma 1].

(ii) We must show that any element $\chi \in C_{\mathbb{Z}}(\mathscr{A})$ is $B$-linear. Indeed if $x, y \in \mathscr{A}$ and $b \in B$ we have $\chi(b \cdot(x y))=\chi(x(b \cdot y))=\chi(x)(b \cdot y)=b \cdot(\chi(x) y)=b \cdot \chi(x y)$.

We now introduce a convenient acronym, pfgc, that will be used throughout the paper.

Definition 2.9. A $B$-algebra $\mathscr{A}$ is said to be $p f g c$ if it satisfies the following conditions

P0. $\mathscr{A} \neq(0)$

P1. $A$ is perfect

P2. $\mathscr{A}$ is finitely generated as a module over its centroid $C_{B}(\mathscr{A})$.

Remark 2.10. The notion of pfgc algebra $\mathscr{A}$ is independent of the base ring under which $\mathscr{A}$ is viewed as an algebra. More precisely, if $\mathscr{A}$ is an algebra over $B$, it follows from Remark 2.7 and Lemma 2.8(ii) that $\mathscr{A}$ is a pfgc algebra over $B$ if and only if $\mathscr{A}$ is a pfgc algebra over $\mathbb{Z}$

We now summarize the basic facts that we will need about pfgc algebras.

Proposition 2.11. Suppose that $\mathscr{A}$ is a pfgc algebra over $B$. Then

(i) $C_{B}(\mathscr{A})$ is a nonzero unital commutative associative $B$-algebra.

(ii) A is finitely generated as a module over its multiplication algebra $\operatorname{Mult}_{B}(\mathscr{A})$.

Proof. (i) Since $\mathscr{A}$ is perfect and nonzero, this follows from Lemma 2.8(i).

(ii) Let $C=C_{B}(\mathscr{A})$. Let $\left\{x_{1}, \ldots, x_{n}\right\} \in \mathscr{A}$ be such that $\mathscr{A}=\sum C x_{i}$. For each $i$ we can write $x_{i}=\sum_{j} y_{i j} z_{i j}$ (finite sum) for some $y_{i j}$ and $z_{i j}$ in $\mathscr{A}$. Then

$$
\mathscr{A}=\sum_{i} C x_{i}=\sum_{i, j} C\left(y_{i j} z_{i j}\right)=\sum_{i, j}\left(C y_{i j}\right) z_{i j} \subset \sum_{i, j} \operatorname{Mult}_{B}(\mathscr{A}) \cdot z_{i j},
$$

which shows that $\mathscr{A}$ is generated by the $z_{i j}$ 's as an $\operatorname{Mult}_{B}(\mathscr{A})$-module. 


\section{Prime pfgc algebras}

In this section, we recall some basic facts about prime algebras and consider in particular properties of prime pfgc algebras. A good basic reference on prime nonassociative algebras and their centroids is [Erickson et al. 1975].

We suppose again in this section that $B$ is a ring and that $\mathscr{A}$ is $B$-algebra.

Definition 3.1. The $B$-algebra $\mathscr{A}$ is said to be prime if for all ideals $\mathscr{I}$ and $\mathscr{F}$ of the $B$-algebra $\mathscr{A}$ we have

$$
\mathscr{I} \mathscr{F}=0 \Longrightarrow \mathscr{I}=0 \text { or } \mathscr{F}=0 .
$$

On the other hand $\mathscr{A}$ is said to be semiprime if for all ideals $\mathscr{I}$ of $\mathscr{A}$ we have

$$
\mathscr{I}=0 \Longrightarrow \mathscr{I}=0 \text {. }
$$

The following lemma which is easily checked (see [Zhevlakov et al. 1982, Exercise $1, \S 8.2]$ ) tells us that the notion of $\mathscr{A}$ being prime (or semiprime) is independent of the base ring under which $\mathscr{A}$ is viewed as an algebra.

Lemma 3.2. A is prime (resp. semiprime) as a B-algebra if and only if $\mathscr{A}$ is prime (resp. semiprime) as a $\mathbb{Z}$-algebra.

The following is proved in [Erickson et al. 1975].

Lemma 3.3. Assume $\mathscr{A}$ is a prime algebra over $B$. Then

(i) $C_{B}(\mathscr{A})$ is an integral domain and $\mathscr{A}$ is a torsion free $C_{B}(\mathscr{A})$-module.

(ii) If we denote the quotient field of $C_{B}(\mathscr{A})$ by $\widetilde{C_{B}(\mathscr{A})}$, then $\mathscr{A} \otimes_{C_{B}(\mathscr{A})} \widetilde{C_{B}(\mathscr{A})}$ is a prime algebra over $\widetilde{C_{B}(\mathscr{A})}$. Moreover, if $\mathscr{A}$ is finitely generated as a module over its multiplication algebra $\operatorname{Mult}_{B}(\mathscr{A})$, then $\mathscr{A} \otimes_{C_{B}(\mathscr{A})} \widetilde{C_{B}(\mathscr{A})}$ is central over $\widetilde{C_{B}(\mathscr{A})}$.

Proof. (i) is Theorem 1.1(a) of [Erickson et al. 1975], whereas (ii) follows from Theorem 1.3(a) and (b) of [Erickson et al. 1975].

In a later section of the paper we will investigate the type of an iterated loop algebra. In that section, we will need the notion of central closure.

Definition 3.4. Let $\mathscr{A}$ be a prime pfgc algebra over $B$. Denote the quotient field of $C_{B}(\mathscr{A})$ by $\widetilde{C_{B}(\mathscr{A})}$, and form the $\widetilde{C_{B}(\mathscr{A})}$-algebra

$$
\tilde{A}:=\mathscr{A} \otimes C_{B}(\mathscr{A}) \widetilde{C_{B}(\mathscr{A})} .
$$

We call $\widetilde{A}$ the central closure of $\mathscr{A}$. (This is not apparently the same as the central closure defined in [Erickson et al. 1975, § II]. Here we are following the terminology in, for example, [McCrimmon and Zel'manov 1988, p. 154].) By Lemma 3.3(i), $\mathscr{A}$ is a torsion free $C_{B}(\mathscr{A})$-module, and so the map $a \mapsto a \otimes 1$ is an injection 
of $\mathscr{A}$ into $\widetilde{A}$ which we regard as an identification. In this way $\mathscr{A}$ is regarded as a subalgebra of its central closure $\tilde{A}$.

We now summarize the main facts that we will need about the central closure:

Proposition 3.5. Let $\mathscr{A}$ be a prime pfgc algebra over $B$. Then the central closure $\widetilde{A}$ of $\mathscr{A}$ is a prime pfgc algebra over $B$. Moreover, $\tilde{A}$ is finite dimensional and central as an algebra over the field $\widetilde{C_{B}(\mathscr{A})}$.

Proof. $\tilde{\mathscr{A}}$ is prime by Lemma 3.3(ii). Next, since $\mathscr{A}$ is embedded as a subalgebra of $\widetilde{A}$, we have $\widetilde{A} \neq 0$. Also, since $\mathscr{A}$ is perfect, $\widetilde{A}$ is perfect. Furthermore, since $\mathscr{A}$ is finitely generated as a $C_{B}(\mathscr{A})$-module, $\tilde{\mathscr{A}}$ is finitely generated as a $\widetilde{C_{B}(\mathscr{A})}$ module and therefore also as a $C_{B}(\widetilde{\mathscr{A}})$-module (since $\lambda_{\widetilde{\mathscr{A}}}\left(\widetilde{C_{B}(\mathscr{A})}\right) \subset C_{B}(\tilde{\mathscr{A}})$ ). Thus $\widetilde{A}$ is pfgc.

We have just seen that $\tilde{\mathscr{A}}$ is finite dimensional over $\widetilde{C_{B}(\mathscr{A})}$.

Finally, since $\mathscr{A}$ is pfgc, Proposition 2.11(ii) tells us that $\mathscr{A}$ is finitely generated as a $\operatorname{Mult}_{B}(\mathscr{A})$-module. Thus $\widetilde{\mathscr{A}}$ is central over $\widetilde{C_{B}(\mathscr{A})}$ by Lemma 3.3(ii).

\section{Loop algebras}

Assumptions and notation: For the rest of the article, $k$ will denote a fixed base field. Unless indicated to the contrary, the term algebra will mean algebra over $k$. For the sake of brevity, if $\mathscr{A}$ is an algebra (over $k$ ), we will often write

$$
C(\mathscr{A}):=C_{k}(\mathscr{A}) \text {. }
$$

In this section we recall the definition of a loop algebra and derive some of its basic properties.

Throughout the section let $m$ be a positive integer and let

$$
\mathbb{Z}_{m}=\{\bar{l}: i \in \mathbb{Z}\}
$$

be the group of integers modulo $m$, where $\bar{\imath}=i+m \mathbb{Z} \in \mathbb{Z}_{m}$ for $i \in \mathbb{Z}$. Let

$$
R=k\left[t^{ \pm 1}\right] \quad \text { and } \quad S=k\left[z^{ \pm 1}\right]
$$

be the algebras of Laurent polynomials in the variables $t$ and $z$ respectively, and we identify $R$ as a subalgebra of $S$ by identifying

$$
t=z^{m} \text {. }
$$

Observe that $S$ is a free $R$-module of rank $m$ with basis $\left\{1, z, \ldots, z^{m-1}\right\}$, and hence the ring extension $S / R$ is faithfully flat.

Recall that a $\mathbb{Z}_{m}$-grading of the algebra $\mathscr{A}$ is an indexed family $\Sigma=\left\{\mathscr{A}_{\bar{l}}\right\}_{\bar{i} \in \mathbb{Z}_{m}}$ of subspaces of $\mathscr{A}$ so that $\mathscr{A}=\bigoplus_{\bar{i} \in \mathbb{Z}_{m}} \mathscr{A}_{\bar{l}}$ and $\mathscr{A}_{\bar{l}} \mathscr{A}_{\bar{j}} \subset \mathscr{A}_{\bar{i}+\bar{j}}$ for $\bar{l}, \bar{j} \in \mathbb{Z}_{m}$. The integer $m$ is called the modulus of $\Sigma$. 
Definition 4.1. Suppose that $\mathscr{A}$ is a $k$-algebra, and we are given a $\mathbb{Z}_{m}$-grading $\Sigma$ of the algebra $\mathscr{A}$ :

$$
\mathscr{A}=\bigoplus_{\bar{\imath} \in \mathbb{Z}_{m}} \mathscr{A}_{\bar{\imath}}
$$

In $\mathscr{A} \otimes_{k} S$ we define

$$
L(\mathscr{A}, \Sigma):=\bigoplus_{i \in \mathbb{Z}} \mathscr{A}_{\bar{l}} \otimes_{k} z^{i}=\left(\mathscr{A}_{\overline{0}} \otimes_{k} R\right) \oplus\left(\mathscr{A}_{\overline{1}} \otimes_{k} z R\right) \oplus \cdots \oplus\left(\mathscr{A}_{\overline{m-1}} \otimes_{k} z^{m-1} R\right) .
$$

Then $L(\mathscr{A}, \Sigma)$ is an $R$-subalgebra of $\mathscr{A} \otimes_{k} S$ that we call the loop algebra of $\Sigma$ based on $\mathscr{A}$. Since $L(\mathscr{A}, \Sigma)$ is an algebra over $R$, it is also an algebra over $k$.

Remark 4.2. If we wish to emphasize the role of the variable $z$ in the construction of the loop algebra we write $L(\mathscr{A}, \Sigma)$ as $L(\mathscr{A}, \Sigma, z)$.

Example 4.3. If $m=1$, then $\mathbb{Z}_{m}=\{\overline{0}\}, \mathscr{A}=\mathscr{A}_{\overline{0}}$ and $L(\mathscr{A}, \Sigma)=\mathscr{A} \otimes_{k} S$ is called the untwisted loop algebra based on $\mathscr{A}$.

Remark 4.4. Suppose that $k$ contains a primitive $m$-th root of unity $\zeta_{m}$. In that case we can choose to work with finite order automorphisms of period $m$ rather than $\mathbb{Z}_{m}$-gradings, provided that we fix the choice of $\zeta_{m}$.

Indeed, suppose that $\mathscr{A}$ is an algebra. If $\sigma$ is an algebra automorphism of period $m$ of $\mathscr{A}$, we may define a $\mathbb{Z}_{m}$-grading $\Sigma=\left\{\mathscr{A}_{\bar{l}}\right\}_{\bar{i} \in \mathbb{Z}_{m}}$ of $\mathscr{A}$ by setting

$$
\mathscr{A}_{\bar{l}}=\left\{x \in \mathscr{A} \mid \sigma(x)=\zeta_{m}^{i} x\right\},
$$

for $\bar{i} \in \mathbb{Z}_{m}$. We refer to this grading $\Sigma$ as the grading determined by $\sigma$. It is clear that any $\mathbb{Z}_{m}$-grading is determined by a unique automorphism $\sigma$ in this way. If $\Sigma$ is the grading determined by $\sigma$, we denote the algebra $L(\mathscr{A}, \Sigma)$ by $L(\mathscr{A}, \sigma)$, or $L(\mathscr{A}, \sigma, z)$ if we want to emphasize the role of $z$. The algebra $L(\mathscr{A}, \sigma)$ can alternately be defined as the subalgebra of fixed points in $\mathscr{A} \otimes_{k} S$ of the automorphism $\sigma \otimes \eta_{m}^{-1}$, where $\eta_{m} \in \operatorname{Aut}_{k}(S)$ is defined by $\eta_{m}(z)=\zeta_{m} z$.

Remark 4.5. When $k=\mathbb{C}, \mathscr{A}$ is a finite dimensional simple Lie algebra over $k$ and $\sigma$ is a finite order automorphism of $\mathscr{A}$, the loop algebra $L(\mathscr{A}, \sigma)$ was used by V. Kac in [Kac 1969] to give realizations of all affine Kac-Moody Lie algebras and to classify finite order automorphisms of $\mathscr{A}$. (See [Kac 1990, Ch. 8] and [Helgason 1978, Ch. X, § 5] for more information about this.)

For the rest of the section, let $\mathscr{A}$ a k-algebra, let $\Sigma$ be a grading of $\mathscr{A}$ by $\mathbb{Z}_{m}$, and let

$$
\mathscr{L}=L(\mathscr{A}, \Sigma) .
$$

We next describe a useful canonical form for elements of $\mathscr{A} \otimes_{k} S$ in terms of elements of $L(\mathscr{A}, \Sigma)$. For this purpose note that $\mathscr{A} \otimes_{k} S$ is an $S$-module (with 
action denoted by ".") and $L(\mathscr{A}, \Sigma)$ is contained in $\mathscr{A} \otimes_{k} S$. Thus we may write expressions like $\sum_{i=0}^{m-1} z^{i} \cdot x_{i} \in \mathscr{A} \otimes_{k} S$ if $x_{0}, \ldots, x_{m-1} \in L(\mathscr{A}, \Sigma)$.

Lemma 4.6. Each element of $\mathscr{A} \otimes_{k} S$ can be written uniquely in the form

$$
\sum_{i=0}^{m-1} z^{i} \cdot x_{i}
$$

where $x_{0}, \ldots, x_{m-1} \in \mathscr{L}$.

Proof. This fact was proved using a Galois cocycle argument in [Allison et al. 2004, Theorem 3.6 (b)] in the case when $k$ contains a primitive $m$-th root of unity. We give a direct proof here instead. Let $x \in \mathscr{A} \otimes_{k} S$. Then $x$ is the sum of elements of the form $a \otimes z^{j}$, where $j \in \mathbb{Z}$ and $a \in \mathcal{A}_{\bar{\ell}}$ for some $\ell \in \mathbb{Z}$. But, if we write $j-\ell=q m+i$, where $q \in \mathbb{Z}$ and $0 \leq i \leq m-1$, then $a \otimes z^{j}=z^{i} \cdot\left(a \otimes z^{j-i}\right)=z^{i} \cdot\left(a \otimes z^{q m+\ell}\right)$ and $a \otimes z^{q m+\ell} \in \mathscr{L}$. So $x$ can be expressed in the form (4-1). For uniqueness, suppose that $\sum_{i=0}^{m-1} z^{i} \cdot x_{i}=0$, where $x_{0}, \ldots, x_{m-1} \in \mathscr{L}$. Write $x_{i}=\sum_{j \in \mathbb{Z}} a_{i j} \otimes z^{j}$, where $a_{i j} \in \mathscr{A}_{\bar{j}}$ for all $j$ and only finitely many $a_{i j}$ are nonzero. Then

$$
\sum_{i=0}^{m-1} \sum_{j \in \mathbb{Z}} a_{i j} \otimes z^{i+j}=0 .
$$

For $0 \leq \ell \leq m-1$, the $A_{\bar{\ell}} \otimes_{k} S$-component of the expression on the left above must be zero. Thus we have

$$
\sum_{i=0}^{m-1} \sum_{j \equiv \ell} a_{i j} \otimes z^{i+j}=0
$$

for $0 \leq \ell \leq m-1$, where $\equiv$ denotes congruence modulo $m$. The exponents $i+j$ appearing in this sum are all distinct and so we have $a_{i j}=0$ for all $i, j$ and hence $x_{i}=0$ for all $i$.

Next note that we have the canonical map $\xi=\xi_{\mathscr{A}, \Sigma}: L(\mathscr{A}, \Sigma) \otimes_{R} S \rightarrow \mathscr{A} \otimes_{k} S$ defined by

$$
\xi\left(x \otimes z^{i}\right)=z^{i} \cdot x
$$

for $x \in L(\mathscr{A}, \Sigma), i \in \mathbb{Z}$. As observed in [Allison et al. 2004, Theorem 3.6(b)], Lemma 4.6 has the following interpretation:

Lemma 4.7. The $\operatorname{map} \xi_{\mathscr{A}, \Sigma}: L(\mathscr{A}, \Sigma) \otimes_{R} S \rightarrow \mathscr{A}_{\otimes_{k}} S$ is an $S$-algebra isomorphism of $L(\mathscr{A}, \Sigma) \otimes_{R} S$ onto $\mathscr{A} \otimes_{k} S$.

Proof. Clearly $\xi$ is a homomorphism of $S$-algebras. Moreover, each element of $L(\mathscr{A}, \Sigma) \otimes_{R} S$ can be expressed in the form $\sum_{i=0}^{m-1} x_{i} \otimes z^{i}$, where $x_{i} \in L(\mathscr{A}, \Sigma)$ for each $i$, and so $\xi$ is bijective by Lemma 4.6. 
Remark 4.8. Lemma 4.7 tells us that after base ring extension from $R$ to $S$ the loop algebra $L(\mathscr{A}, \Sigma)$ becomes isomorphic to the untwisted loop algebra $\mathscr{A} \otimes_{k} S$. In other words, $L(\mathscr{A}, \Sigma)$ is "untwisted" by the extension $S / R$. This fact is of great importance in the study of loop algebras since, among other things, it allows one to use the tools of Galois cohomology to study loop algebras [Allison et al. 2004; Pianzola 2002].

\section{Lemma 4.9.}

(i) If $\mathscr{A} \neq 0$, then $L(\mathscr{A}, \Sigma) \neq 0$.

(ii) If $\mathscr{A}$ is perfect, then $L(\mathscr{A}, \Sigma)$ is perfect.

Proof. Statement (i) is clear and statement (ii) is easily checked (see the argument in [Allison et al. 2004, Lemma 4.3]).

We now examine the centroid of $\mathscr{L}=L(\mathscr{A}, \Sigma)$.

First note that since $\mathscr{L}$ is an $R$-algebra, $C_{R}(\mathscr{L})$ is naturally an $R$-algebra (see Definition 2.1(iii)). So since $C_{R}(\mathscr{L}) \subset C(\mathscr{L})$, it follows that $C(\mathscr{L})$ is also an $R$ algebra.

Next by Lemma 2.5 the centroid $C(\mathscr{A})$ inherits a $\mathbb{Z}_{m}$-grading that we denote by $C(\Sigma)$. Under this grading we have

where

$$
C(\mathscr{A})=\bigoplus_{\bar{\imath} \in \mathbb{Z}_{m}} C(\mathscr{A})_{\bar{\imath}}
$$

$$
C(\mathscr{A})_{\bar{\imath}}=\left\{\chi \in C(\mathscr{A}) \mid \chi\left(\mathscr{A}_{\bar{j}}\right) \subset \mathscr{A}_{\bar{\imath}+\bar{j}} \text { for } \bar{j} \in \mathbb{Z}_{m}\right\} .
$$

Now let

$$
\psi:=\psi_{\mathscr{A}, \Sigma}: L(C(\mathscr{A}), C(\Sigma)) \rightarrow C_{R}(L(\mathscr{A}, \Sigma))
$$

be the unique $k$-linear map so that

$$
\left(\psi\left(\chi \otimes z^{i}\right)\right)\left(a \otimes z^{j}\right)=\chi(a) \otimes z^{i+j}
$$

for $i, j \in \mathbb{Z}, \chi \in C(\mathscr{A})_{\bar{l}}, a \in \mathscr{A}_{\bar{\jmath}}$. It is immediate from this definition that $\psi$ is a homomorphism of $R$-algebras that we call canonical.

Lemma 4.10. Assume $\mathscr{A}$ is finitely generated as a module over its multiplication algebra $\operatorname{Mult}_{k}(\mathscr{A})$. Then the map $\psi_{\mathscr{A}, \Sigma}: L(C(\mathscr{A}), C(\Sigma)) \rightarrow C_{R}(L(\mathscr{A}, \Sigma))$ is an $R$-algebra isomorphism.

Proof. Since the ring extension $S / R$ is faithfully flat, to show that $\psi$ is an $R$-module isomorphism it suffices to show that $\psi$ becomes an isomorphism of $S$-modules after the base change from $R$ to $S$. That this is so follows from the commutative diagram 


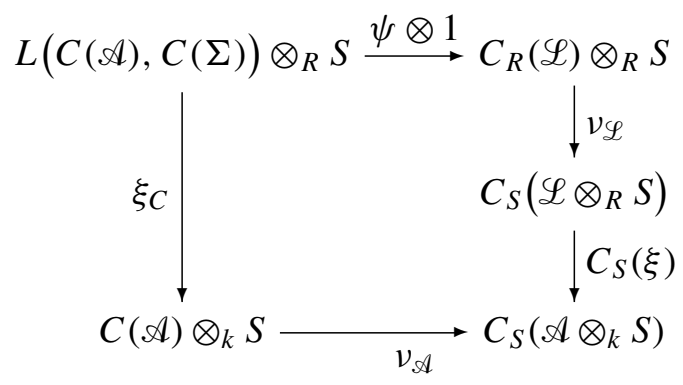

in view of the fact that all vertical maps and the bottom row therein are $S$-isomorphisms. In this diagram $\xi_{C}=\xi_{C(\mathscr{A}), C(\Sigma)}$ as in Lemma 4.7, $v_{\mathscr{A}}=v_{\mathscr{A}, k, S}$ as in Lemma 2.3(a), $v_{\mathscr{L}}=v_{\mathscr{L}, R, S}$ as in Lemma 2.3(b), and $C_{S}(\xi)$ is the isomorphism induced by the isomorphism $\xi=\xi_{\mathscr{A}, \Sigma}: \mathscr{L} \otimes_{R} S \rightarrow \mathscr{A} \otimes_{k} S$ (see Lemmas 2.2 and 4.7).

The following proposition tells us that the centroid of a loop algebra based on a pfgc algebra $\mathscr{A}$ is isomorphic to the loop algebra of the centroid of $\mathscr{A}$.

Proposition 4.11. Let $\mathscr{L}=L(\mathscr{A}, \Sigma)$ be a loop algebra based on a pfgc algebra $A$. Then $C_{R}(\mathscr{L})=C(\mathscr{L})$, and the canonical map

$$
\psi=\psi_{\mathscr{A}, \Sigma}: L(C(\mathscr{A}), C(\Sigma)) \rightarrow C(\mathscr{L})
$$

is an R-algebra isomorphism.

Proof. Since $\mathscr{L}$ is perfect by Lemma 4.9(ii), it follows that $C_{R}(\mathscr{L})=C(\mathscr{L})$ by Lemma 2.8(ii). Also since $\mathscr{A}$ is pfgc, it follows from Proposition 2.11(ii) that $\mathscr{A}$ is finitely generated as a module over $\operatorname{Mult}_{k}(\mathscr{A})$. Thus, by Lemma $4.10, \psi$ is an $R$-algebra isomorphism from $L(C(\mathscr{A}), C(\Sigma))$ onto $C(\mathscr{L})$.

Finally we want to show that a loop algebra based on a pfgc algebra is pfgc. For this we will use the following:

Lemma 4.12. If $\mathscr{A}$ is finitely generated as a $C(\mathscr{A})$-module then $L(\mathscr{A}, \Sigma)$ is finitely generated as a $C_{R}(L(\mathscr{A}, \Sigma))$-module.

Proof. Let $\left\{a_{1}, \ldots, a_{p}\right\}$ be a set of homogeneous elements of $\mathscr{A}$ that generates $\mathscr{A}$ as a $C(\mathscr{A})$-module. Fix integers $d_{1}, \ldots, d_{p}$ so that $a_{j} \in \mathscr{A}_{\overline{d_{j}}}$. Let $M$ be the $C_{R}(\mathscr{L})$-submodule of $\mathscr{L}$ generated by the elements $a_{k} \otimes z^{d_{k}}$. Since $S / R$ is flat we may identify $M \otimes_{R} S$ as an $S$-submodule of $\mathscr{L} \otimes_{R} S$, and since $S / R$ is faithfully flat it is sufficient to show that $\mathcal{M} \otimes_{R} S=\mathscr{L} \otimes_{R} S$ [Bourbaki 1972, Ch. I, §3.1, Proposition 2]. We do this by showing that $\xi\left(M \otimes_{R} S\right)=\xi\left(\mathscr{L} \otimes_{R} S\right)$, where $\xi=$ $\xi_{A, \Sigma}: \mathscr{L} \otimes_{R} S \longrightarrow \mathscr{A} \otimes_{k} S$ is the $S$-algebra isomorphism from Lemma 4.7.

Suppose that $i, j \in \mathbb{Z}, \chi \in C(\mathscr{A})_{\bar{i}}$ and $1 \leq \ell \leq p$. Then $\psi\left(\chi \otimes z^{i}\right)$ is an element of $C_{R}(\mathscr{L})$, where $\psi=\psi_{\mathscr{A}, \Sigma}$. So $\left(\psi\left(\chi \otimes z^{i}\right)\right)\left(a_{\ell} \otimes z^{d_{\ell}}\right) \in \mathcal{M}$. But under $\xi$ we have

$$
\left(\left(\psi\left(\chi \otimes z^{i}\right)\right)\left(a_{\ell} \otimes z^{d_{\ell}}\right)\right) \otimes z^{j} \mapsto \chi\left(a_{\ell}\right) \otimes z^{d_{\ell}+i+j} .
$$


Since $\left\{a_{1}, \ldots, a_{p}\right\}$ generates $\mathscr{A}$ as a $C(\mathscr{A})$-module, it follows $\xi\left(M \otimes_{R} S\right)=\xi\left(\mathscr{L} \otimes_{R}\right.$ $S)$ as needed.

Proposition 4.13. Let $\mathscr{L}=L(\mathscr{A}, \Sigma)$ be a loop algebra based on a pfgc algebra $A$. Then $\mathscr{L}$ is a pfgc algebra.

Proof. $\mathscr{L} \neq(0)$ and $\mathscr{L}$ is perfect by Lemma 4.9. So P0 and P1 hold (see Definition 2.9). By Lemma $4.12, \mathscr{L}$ is finitely generated as a $C_{R}(\mathscr{L})$-module. But by Proposition 4.11, we have $C_{R}(\mathscr{L})=C(\mathscr{L})$. Thus $\mathscr{L}$ is finitely generated as a $C(\mathscr{L})$-module and so $\mathrm{P} 2$ holds. Hence $\mathscr{L}$ is pfgc.

\section{Iterated loop algebras}

In this section we define iterated loop algebras and prove some of their basic properties.

Notation: For the rest of this article, we fix some notation. Let $n$ be a positive integer. Let $z_{1}, \ldots, z_{n}$ be a sequence of algebraically independent variables over $k$. For $0 \leq p \leq n$, let

$$
S^{\otimes p}:=k\left[z_{1}^{ \pm 1}, \ldots, z_{p}^{ \pm 1}\right]
$$

be the algebra of Laurent polynomials in the variables $z_{1}, \ldots, z_{p}$ over $k$. (So $S^{\otimes 0}=k$.) We identify $S^{\otimes p} \otimes S^{\otimes q}=S^{\otimes(p+q)}$ in the natural fashion when $0 \leq p, q \leq n$ and $p+q \leq n$. We also fix a sequence $m_{1}, \ldots, m_{n}$ of positive integers, and we set

$$
I_{p}:=\left\{\left(i_{1}, \ldots, i_{p}\right) \in \mathbb{Z}^{p} \mid 0 \leq i_{j} \leq m_{j}-1 \text { for all } j\right\},
$$

for $1 \leq p \leq n$.

Definition 5.1. Suppose that $\mathscr{A}$ is an algebra over $k$. An algebra $\mathscr{L}$ over $k$ is called an $n$-step loop algebra or an iterated loop algebra based on $\mathscr{A}$ if there exists a sequence $\mathscr{L}_{0}, \mathscr{L}_{1}, \ldots, \mathscr{L}_{n}$ of algebras so that $\mathscr{L}_{0}=\mathscr{A}, \mathscr{L}_{n}=\mathscr{L}$ and

$$
\mathscr{L}_{p}=L\left(\mathscr{L}_{p-1}, \Sigma_{p}, z_{p}\right),
$$

for $1 \leq p \leq n$, where $\Sigma_{p}$ is a $\mathbb{Z}_{m_{p}}$-grading of $\mathscr{L}_{p-1}$. (See Remark 4.2 for the notation used here.) In that case we write

$$
\mathscr{L}=L\left(\mathscr{A}, \Sigma_{1}, \ldots, \Sigma_{n}\right)
$$

(suppressing in the notation the role of the variables $z_{1}, \ldots, z_{n}$ ).

Remark 5.2. Suppose that $\mathscr{L}$ is an $n$-step loop algebra based on $\mathscr{A}$ and we have the notation from Definition 5.1.

(i) For $1 \leq p \leq n, \mathscr{L}_{p}=L\left(\mathscr{A}, \Sigma_{1}, \ldots, \Sigma_{p}\right)$ is a $p$-step loop algebra based on $\mathscr{A}$.

(ii) Observe that $\mathscr{L}_{p} \subset \mathscr{L}_{p-1} \otimes_{k} k\left[z_{p}^{ \pm 1}\right]$ for $1 \leq p \leq n$. Thus

$$
\mathscr{L}_{p} \subset\left(\ldots\left(\left(\mathscr{A} \otimes_{k} k\left[z_{1}^{ \pm 1}\right]\right) \otimes_{k} k\left[z_{2}^{ \pm 1}\right]\right) \ldots\right) \otimes_{k} k\left[z_{p}^{ \pm 1}\right]=\mathscr{A} \otimes k\left[z_{1}^{ \pm 1}, \ldots, z_{p}^{ \pm 1}\right],
$$


for $0 \leq p \leq n$, where the last equality is the natural identification using the associativity of the tensor product and the identification $k\left[z_{1}^{ \pm 1}\right] \otimes_{k} \ldots \otimes_{k} k\left[z_{p}^{ \pm 1}\right]=S^{\otimes p}$. Consequently, $\mathscr{L}_{p}$ is a subalgebra of $\mathscr{A}_{\otimes_{k}} S^{\otimes p}$ for $0 \leq p \leq n$, and in particular $\mathscr{L}$ is a subalgebra of $\mathscr{A} \otimes_{k} S^{\otimes n}$.

(iii) Suppose that $k$ contains a primitive $m_{i}$-th root of unity $\zeta_{m_{i}}$ (which we fix) for $i=1, \ldots, n$. Then for $p=1, \ldots, n$, the grading $\Sigma_{p}$ of $\mathscr{L}_{p-1}$ is determined by a unique automorphism $\sigma_{p}$ of $\mathscr{L}_{p-1}$ of period $m_{p}$. We then denote the algebra $L\left(\mathscr{A}, \Sigma_{1}, \ldots, \Sigma_{n}\right)$ by $L\left(\mathscr{A}, \sigma_{1}, \ldots, \sigma_{n}\right)$.

Example 5.3. If $m_{1}=\cdots=m_{n}=1$ then $L\left(\mathscr{A}, \Sigma_{1}, \ldots, \Sigma_{n}\right)=\mathscr{A} \otimes_{k} S^{\otimes n}$ is called the untwisted $n$-step loop algebra based on $\mathscr{A}$.

Example 5.4 (Multiloop algebras). Suppose that $k$ contains a primitive $m_{i}$-th root of unity $\zeta_{m_{i}}$ for $1 \leq i \leq n$. Let $\mathscr{A}$ be an algebra, and let $\sigma_{1}, \ldots, \sigma_{n}$ be commuting finite order automorphisms of $\mathscr{A}$ with periods $m_{1}, \ldots, m_{n}$ respectively. Let

$$
\mathscr{A}_{\bar{l}_{1}, \ldots, \bar{l}_{n}}=\left\{x \in \mathscr{A} \mid \sigma_{j} x=\zeta_{m_{j}}^{i_{j}} x \text { for } 1 \leq j \leq n\right\}
$$

for $\left(i_{1}, \ldots, i_{n}\right) \in \mathbb{Z}^{n}$, where $\bar{l}_{j}:=i_{j}+m_{j} \mathbb{Z} \in \mathbb{Z}_{m_{j}}$ for $1 \leq j \leq n$. Then

$$
\mathscr{A}=\bigoplus_{\left(i_{1}, \ldots, i_{n}\right) \in I_{n}} \mathscr{A}_{\bar{l}_{1}, \ldots, \bar{i}_{n}},
$$

and we set

$$
M\left(\mathscr{A}, \sigma_{1}, \ldots, \sigma_{n}\right):=\bigoplus_{\left(i_{1}, \ldots, i_{n}\right) \in \mathbb{Z}^{n}} A_{\bar{i}_{1}, \ldots, \bar{i}_{n}} \otimes_{k} z_{1}^{i_{1}} \ldots z_{n}^{i_{n}}
$$

in $\mathscr{A} \otimes_{k} S^{\otimes n}$. Then $M\left(\mathscr{A}, \sigma_{1}, \ldots, \sigma_{n}\right)$ is a subalgebra of $\mathscr{A} \otimes_{k} S^{\otimes n}$ that we call the $n$-step multiloop algebra of $\sigma_{1}, \ldots, \sigma_{n}$ based on $\mathscr{A}$.

Now the multiloop algebra $\mathscr{L}=M\left(\mathscr{A}, \sigma_{1}, \ldots, \sigma_{n}\right)$ can be interpreted as an iterated loop algebra. To see this, let $\mathscr{L}_{0}=\mathscr{A}$ and let $\mathscr{L}_{p}=M\left(\mathscr{A}, \sigma_{1}, \ldots, \sigma_{p}\right)$ for $1 \leq p \leq n$. Then by definition we have $\mathscr{L}_{0}=\mathscr{A}$ and $\mathscr{L}_{n}=\mathscr{L}$. Also, for $1 \leq p \leq n$, we may define a $\mathbb{Z}_{m_{p}}$-grading $\Sigma_{p}$ on $\mathscr{L}_{p-1}$ by setting

$$
\left(\mathscr{L}_{p-1}\right)_{\bar{l}_{p}}=\bigoplus_{\left(i_{1}, \ldots, i_{p-1}\right) \in \mathbb{Z}^{p-1}} \mathscr{A}_{\bar{l}_{1}, \ldots, \bar{l}_{p}} \otimes_{k} z_{1}^{i_{1}} \ldots z_{p-1}^{i_{p-1}}
$$

for $\bar{\imath}_{p} \in \mathbb{Z}_{m_{p}}$, in which case it is then clear that $L\left(\mathscr{L}_{p-1}, \Sigma_{p}, z_{p}\right)=\mathscr{L}_{p}$. So $\mathscr{L}=$ $L\left(\mathscr{A}, \Sigma_{1}, \ldots, \Sigma_{n}\right)$.

We have just seen in Example 5.4 that any multiloop algebra is an iterated loop algebra. However we will see later in Example 9.7 that there are iterated loop algebras $\mathscr{A}$ that are not multiloop algebras.

For the rest of the section we assume that

$$
\mathscr{L}=L\left(\mathscr{A}, \Sigma_{1}, \ldots, \Sigma_{n}\right)
$$


is an n-step loop algebra based on an algebra $\mathscr{A}$ over $k$, and we use the notation $\mathscr{L}_{0}, \ldots, \mathscr{L}_{n}$ of Definition 5.1.

Our first theorem describes some important basic algebraic properties that are inherited by a loop algebra from its base. In the last part of this theorem we will see how the Krull dimension of a loop algebra depends on the Krull dimension of its base. Here and subsequently we use

\section{$\operatorname{Dim} \mathscr{C}$}

to denote the Krull dimension of a unital commutative associative $k$-algebra $\mathscr{C}$ (when regarded as a ring). Note that if $\mathscr{C}$ is finitely generated as a $k$-algebra then $\operatorname{Dim} \mathscr{C}$ is finite [Kunz 1985, p. 52].

Theorem 5.5. Let $\mathscr{L}=L\left(\mathscr{A}, \Sigma_{1}, \ldots, \Sigma_{n}\right)$.

(i) If $\mathscr{A} \neq 0$ then $\mathscr{L} \neq 0$.

(ii) If $\mathscr{A}$ is perfect then $\mathscr{L}$ is perfect.

(iii) If $\mathscr{A}$ is pfgc then $\mathscr{L}$ is pfgc.

(iv) If $\mathscr{A}$ is prime then $\mathscr{L}$ is prime.

(v) If $\mathscr{A}$ is unital then $\mathscr{L}$ is a unital subalgebra of $\mathscr{A} \otimes S^{\otimes n}$.

(vi) If $\mathscr{A}$ is commutative then $\mathscr{L}$ is commutative.

(vii) If $\mathscr{A}$ is associative then $\mathscr{L}$ is associative.

(viii) If $\mathscr{A}$ is an integral domain then $\mathscr{L}$ is an integral domain.

(ix) If $\mathscr{A}$ is unital and finitely generated as a $k$-algebra then $\mathscr{L}$ is unital and finitely generated as a k-algebra.

(x) If $A$ is unital, commutative, associative and finitely generated as a k-algebra, then $\mathscr{L}$ has the same properties and

$$
\operatorname{Dim} \mathscr{L}=\operatorname{Dim} \mathscr{A}+n .
$$

Proof. Since $\mathscr{L}_{p+1}$ is a loop algebra based on $\mathscr{L}_{p}$ for $0 \leq p \leq n-1$, we can assume in the proof of each of these statements that $n=1$. So we may use the notation of Section 4:

$m=m_{1}, \quad z=z_{1}, \quad \Sigma=\Sigma_{1}, \mathscr{L}=L(\mathscr{A}, \Sigma, z), S=S^{\otimes 1}=k\left[z^{ \pm 1}\right]$ and $R=k\left[z^{ \pm m}\right]$.

Now (i) and (ii) follow from Lemma 4.9. (iii) follows from Proposition 4.13. (v) follows from that fact that $1_{\mathscr{A}} \in \mathscr{A}_{\overline{0}}$, since then $1_{\mathscr{A}} \otimes 1_{S} \in \mathscr{L}$. (vi), (vii) and (viii) follow from the fact that $\mathscr{L}$ is a subalgebra of $\mathscr{A} \otimes_{k} S \simeq_{k} \mathscr{A}\left[z^{ \pm 1}\right]$. So we only need to prove (iv), (ix) and (x). 
(iv) We show first that $\mathscr{A} \otimes_{k} S$ is prime. For this let $\mathscr{I}$ and $\mathscr{S}$ be ideals of the $k$-algebra $\mathscr{A} \otimes_{k} S$ such that $\mathscr{g}=0$. For $m \in \mathbb{Z}$, let

$$
\mathscr{I}_{m}=\left\{a \in \mathscr{A} \mid \exists a_{i} \in \mathscr{A} \text { for } i \geq m \text { with } a_{m}=a \text { and } \sum_{i \geq m} a_{i} \otimes z^{i} \in \mathscr{I}\right\},
$$

in which case $\mathscr{I}_{m}$ is an ideal of $\mathscr{A}$. Similarly, using $\mathscr{I}$ instead of $\mathscr{I}$, we define an ideal $\Phi_{n}$ of $\mathscr{A}$ for $n \in \mathbb{Z}$. Furthermore, since $\mathscr{I} \mathscr{\mathscr { S }}=0$, we have $\Phi_{m} \Phi_{n}=0$ for $m, n \in \mathbb{Z}$. Now suppose that $\mathscr{I} \neq 0$. Then $\Phi_{m} \neq 0$ for some $m \in \mathbb{Z}$. Thus, since $\mathscr{A}$ is prime, we have $\mathscr{F}_{n}=0$ for all $n \in \mathbb{Z}$ and so $\mathscr{E}=0$. Therefore $\mathscr{A}_{k} S$ is prime.

But $\mathscr{L} \otimes_{R} S \simeq_{S} \mathscr{A} \otimes_{k} S$ by Lemma 4.7. Hence $\mathscr{L} \otimes_{R} S$ is a prime algebra. To prove that $\mathscr{L}$ is prime (as a $k$-algebra), it is enough to show that $\mathscr{L}$ is a prime $R$ algebra (by Lemma 3.2). For this let $\mathscr{I}$ and $\mathscr{I}$ be ideals of the $R$-algebra $\mathscr{L}$ such

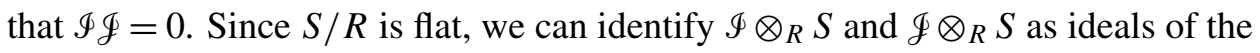
$S$-algebra $\mathscr{L} \otimes_{R} S$. Furthermore, we have $\left(\mathscr{I} \otimes_{R} S\right)\left(\mathscr{F} \otimes_{R} S\right)=0$. Since $\mathscr{L} \otimes_{R} S$ is prime, either $\mathscr{I} \otimes_{R} S$ or $\mathscr{G} \otimes_{R} S$ is 0 . Therefore $\mathscr{I}=0$ or $\mathscr{F}=0$ by the faithful flatness of $S / R$.

(ix) $\mathscr{L}$ is unital by (v). Let $\left\{a_{1}, \ldots, a_{p}\right\}$ be a set of homogeneous elements of $\mathscr{A}$ that generates $\mathscr{A}$ as a $k$-algebra, and fix integers $d_{1}, \ldots, d_{p}$ so that $a_{j} \in \mathscr{A}_{\bar{d}_{j}}$. One easily checks that the elements $a_{1} \otimes z^{d_{1}}, \ldots, a_{p} \otimes z^{d_{p}}$ together with the elements $1_{\mathscr{A}} \otimes z^{m}$ and $1_{\mathscr{A}} \otimes z^{-m}$ generate $\mathscr{L}$ as a $k$-algebra.

(x) $\mathscr{L}$ is unital, commutative, associative and finitely generated as a $k$-algebra by (v), (vi), (vii) and (ix), and so the Krull dimensions of both $\mathscr{A}$ and $\mathscr{L}$ are finite. Now recall that $\mathscr{L}$ is a subalgebra of $\mathscr{A} \otimes_{k} S$ and, by Lemma 4.6, each element of $\mathscr{A} \otimes_{k} S$ can be written uniquely in the form

$$
\sum_{i=0}^{m-1} z^{i} \cdot x_{i}=\sum_{i=0}^{m-1} x_{i}\left(1_{A} \otimes z^{i}\right)
$$

where $x_{0}, \ldots, x_{m-1} \in \mathscr{L}$. Thus $\mathscr{A} \otimes_{k} S$ is a free $\mathscr{L}$-module of rank $m$, and so in particular $\mathscr{A} \otimes_{k} S$ is a finitely generated $\mathscr{L}$-module. Hence $\mathscr{A} \otimes_{k} S / \mathscr{L}$ is an integral ring extension and so by [Kunz 1985, Corollary II.2.13],

$$
\operatorname{Dim} \mathscr{L}=\operatorname{Dim}\left(\mathscr{A} \otimes_{k} S\right) .
$$

On the other hand since both $\mathscr{A}$ and $S$ are finitely generated $k$-algebras

$$
\operatorname{Dim}\left(\mathscr{A} \otimes_{k} S\right)=\operatorname{Dim} \mathscr{A}+\operatorname{Dim} S
$$

[Kunz 1985, Corollary II.3.9]. Since $\operatorname{Dim} S=1$, we obtain $\operatorname{Dim} \mathscr{L}=\operatorname{Dim} \mathscr{A}+1$.

Remark 5.6. It follows in particular from Theorem 5.5 that any $n$-step loop algebra based on a prime pfgc algebra is a prime pfgc algebra. The corresponding statement is not true for simple pfgc algebras. For example, an untwisted pfgc algebra $A_{A} \otimes_{k}$ $S^{\otimes n}$ is never simple (since $S^{\otimes n}$ is not simple). This is the reason why prime pfgc 
algebras are natural algebras to consider when studying loop algebras, even if one's main interest is in the case when the base algebras are simple.

We conclude this section with a generalization to iterated loop algebras of the canonical form described in Lemma 4.6. If $1 \leq p \leq n$, we use the usual convenient notation

$$
z^{\mathbf{i}}=z_{1}^{i_{1}} \ldots z_{p}^{i_{p}}
$$

for $\mathbf{i}=\left(i_{1}, \ldots, i_{p}\right) \in \mathbb{Z}^{p}$. Note that $\mathscr{A} \otimes_{k} S^{\otimes p}$ is an $S^{\otimes p}$-module (with action denoted by “."), and $\mathscr{L}_{p}$ is contained in $\mathscr{A} \otimes_{k} S^{\otimes p}$. Thus, we can write expressions like $\sum_{\mathbf{i} \in I_{p}} z^{\mathbf{i}} \cdot x_{\mathbf{i}} \in \mathscr{A} \otimes_{k} S^{\otimes p}$, where $x_{\mathbf{i}} \in \mathscr{L}_{p}$ for all $\mathbf{i} \in I_{p}$.

Lemma 5.7. If $1 \leq p \leq n$, each element in $A_{A} \otimes_{k} S^{\otimes p}$ can be expressed uniquely in the form

$$
\sum_{\mathbf{i} \in I_{p}} z^{\mathbf{i}} \cdot x_{\mathbf{i}}
$$

where $x_{\mathbf{i}} \in \mathscr{L}_{p}$ for all $\mathbf{i}$.

Proof. We argue by induction on $p$. When $p=1$, the statement follows from Lemma 4.6. So we suppose that the statement is true for $p$, where $1 \leq p \leq n-1$.

Let $x \in \mathscr{A} \otimes S^{\otimes(p+1)}$. To show that $x$ can be expressed in the form (5-2), note first that $x$ is a sum of elements of the form $x^{\prime} \otimes z_{p+1}^{j}$, where $x^{\prime} \in \mathscr{A} \otimes_{k} S^{\otimes p}$ and $j \in \mathbb{Z}$. But by the induction hypothesis, $x^{\prime}$ is the sum of elements of the form $z^{\mathbf{i}} \cdot x^{\prime \prime}$, where $\mathbf{i} \in I_{p}$ and $x^{\prime \prime} \in \mathscr{L}_{p}$. Thus $x$ is the sum of elements of the form

$$
\left(z^{\mathbf{i}} \cdot x^{\prime \prime}\right) \otimes z_{p+1}^{j}=z^{\mathbf{i}} \cdot\left(x^{\prime \prime} \otimes z_{p+1}^{j}\right) .
$$

But $x^{\prime \prime} \otimes z_{p+1}^{j} \in \mathscr{L}_{p} \otimes_{k} k\left[z_{p+1}^{ \pm 1}\right]$, and so, by Lemma 4.6, $x^{\prime \prime} \otimes z_{p+1}^{j}$ is the sum of elements of the form $z_{p+1}^{\ell} \cdot x^{\prime \prime \prime}$, where $0 \leq \ell \leq m_{p+1}-1$ and $x^{\prime \prime \prime} \in \mathscr{L}_{p+1}$. Thus $x$ is the sum of elements of the form

$$
z^{\mathbf{i}} \cdot\left(z_{p+1}^{\ell} \cdot x^{\prime \prime \prime}\right)=\left(z^{\mathbf{i}} z_{p+1}^{\ell}\right) \cdot x^{\prime \prime \prime}
$$

as desired.

For uniqueness, suppose that $\sum_{\mathbf{j} \in I_{p+1}} z^{\mathbf{j}} \cdot x_{\mathbf{j}}=0$, where $x_{\mathbf{j}} \in \mathscr{L}_{p+1}$ for each $\mathbf{j} \in I_{p+1}$. Then

$$
\sum_{\mathbf{i} \in I_{p}} \sum_{\ell=0}^{m_{p+1}-1}\left(z^{\mathbf{i}} z_{p+1}^{\ell}\right) \cdot x_{\mathbf{i}, \ell}=0
$$


where, if $\mathbf{i}=\left(i_{1}, \ldots, i_{p}\right) \in I_{p}$ and $0 \leq \ell \leq m_{p+1}-1$, we are using the notation $x_{\mathbf{i}, \ell}:=x_{\left(i_{1}, \ldots, i_{p}, \ell\right)} \in \mathscr{L}_{p+1}$. So we have

$$
\sum_{\mathbf{i} \in I_{p}} z^{\mathbf{i}} \cdot\left(\sum_{\ell=0}^{m_{p+1}-1} z_{p+1}^{\ell} \cdot x_{\mathbf{i}, \ell}\right)=0 .
$$

But for $\mathbf{i} \in I_{p}$, the element $\sum_{\ell=0}^{m_{p+1}-1} z_{p+1}^{\ell} \cdot x_{\mathbf{i}, \ell}$ is in $\mathscr{L}_{p} \otimes_{k} k\left[z_{p+1}^{ \pm 1}\right]$ and therefore we can write

$$
\sum_{\ell=0}^{m_{p+1}-1} z_{p+1}^{\ell} \cdot x_{\mathbf{i}, \ell}=\sum_{j \in \mathbb{Z}} y_{\mathbf{i}, j} \otimes z_{p+1}^{j},
$$

where each $y_{\mathbf{i}, j}$ is in $\mathscr{L}_{p}$ and only finitely many of these elements are nonzero. Then $\sum_{\mathbf{i} \in I_{p}} z^{\mathbf{i}} \cdot\left(\sum_{j \in \mathbb{Z}} y_{\mathbf{i}, j} \otimes z_{p+1}^{j}\right)=0$, and so

$$
\sum_{j \in \mathbb{Z}}\left(\sum_{\mathbf{i} \in I_{p}} z^{\mathbf{i}} \cdot y_{\mathbf{i}, j}\right) \otimes z_{p+1}^{j}=0 .
$$

Hence $\sum_{\mathbf{i} \in I_{p}} z^{\mathbf{i}} \cdot y_{\mathbf{i}, j}=0$ for each $j$ and so by the induction hypothesis $y_{\mathbf{i}, j}=0$ for all $\mathbf{i} \in I_{p}$ and $j \in \mathbb{Z}$. So $\sum_{\ell=0}^{m_{p+1}-1} z_{p+1}^{\ell} \cdot x_{\mathbf{i}, \ell}=0$ for all $\mathbf{i} \in I_{p}$, and hence, by Lemma 4.6, $x_{\mathbf{i}, \ell}=0$ for all $\mathbf{i} \in I_{p}$ and $0 \leq \ell \leq m_{p+1}-1$.

If $\mathscr{A}$ is unital and associative, then $\mathscr{L}=L\left(\mathscr{A}, \Sigma_{1}, \ldots, \Sigma_{n}\right)$ is a unital associative subalgebra of $\mathscr{A} \otimes_{k} S^{\otimes n}$ and hence $\mathscr{A} \otimes_{k} S^{\otimes n}$ is an $\mathscr{L}$-module (with action denoted by “.”).

Corollary 5.8. Suppose that $\mathscr{L}=L\left(\mathscr{A}, \Sigma_{1}, \ldots, \Sigma_{n}\right)$ where $\mathscr{A}$ is unital and associative. Then $\mathscr{A} \otimes_{k} S^{\otimes n}$ is a free $\mathscr{L}$-module of rank $m_{1} \ldots m_{n}$ with basis

$$
\left\{1_{\mathscr{A}} \otimes z^{\mathbf{i}}\right\}_{\mathbf{i} \in I_{n}} .
$$

Proof. This follows from Lemma 5.7 (with $p=n$ ) and the observation that

$$
z^{\mathbf{i}} \cdot x=x \cdot\left(1_{\mathscr{A}} \otimes z^{\mathbf{i}}\right)
$$

for $x \in \mathscr{L}$ and $\mathbf{i} \in \mathbb{Z}^{n}$. (On the left of this equation · denotes the action of $S^{\otimes n}$ on $\mathscr{A} \otimes_{k} S^{\otimes n}$, whereas on the right $\cdot$ denotes the action of $\mathscr{L}$ on $\mathscr{A} \otimes_{k} S^{\otimes n}$.)

\section{The centroid of an iterated loop algebra}

In this section, we give an explicit description of the centroid of an $n$-step loop algebra based on a pfgc algebra $\mathscr{A}$ as an $n$-step loop algebra based on $C(\mathscr{A})$.

Throughout the section we assume that $\mathscr{L}=L\left(\mathscr{A}, \Sigma_{1}, \ldots, \Sigma_{n}\right)$ is an n-step loop algebra based on a algebra $\mathscr{A}$ over $k$. So we have algebras $\mathscr{L}_{0}, \ldots, \mathscr{L}_{n}$ so 
that $\mathscr{L}_{0}=\mathscr{A}, \mathscr{L}_{n}=\mathscr{L}$ and

$$
\mathscr{L}_{p+1}=L\left(\mathscr{L}_{p}, \Sigma_{p+1}, z_{p+1}\right)
$$

for $0 \leq p \leq n-1$. As we observed in Remark 5.2, $\mathscr{L}_{p}$ is a subalgebra of $\mathscr{A} \otimes_{k} S^{\otimes p}$ for $0 \leq p \leq n$.

We next introduce some notation.

First let $0 \leq p \leq n$. Then $C(\mathscr{A}) \otimes_{k} S^{\otimes p}$ is a unital associative algebra and $\mathscr{A} \otimes_{k} S^{\otimes p}$ is a $C(\mathscr{A}) \otimes_{k} S^{\otimes p}$-module under the action "." defined by

$$
\left(\chi \otimes z^{\mathbf{i}}\right) \cdot\left(a \otimes z^{\mathbf{j}}\right)=\chi(a) \otimes z^{\mathbf{i}+\mathbf{j}} .
$$

We let $\bar{C}\left(\mathscr{L}_{p}\right)$ denote the stabilizer of $\mathscr{L}_{p}$ in $C(\mathscr{A}) \otimes_{k} S^{\otimes p}$ under this action. That is we let

$$
\bar{C}\left(\mathscr{L}_{p}\right):=\left\{u \in C(\mathscr{A}) \otimes_{k} S^{\otimes p} \mid u \cdot \mathscr{L}_{p} \subset \mathscr{L}_{p}\right\} .
$$

Then $\bar{C}\left(\mathscr{L}_{p}\right)$ is a unital subalgebra of $C(\mathscr{A}) \otimes_{k} S^{\otimes p}$ and $\mathscr{L}_{p}$ is a $\bar{C}\left(\mathscr{L}_{p}\right)$-module. (For convenience, our notation suppresses the fact that $\bar{C}\left(\mathscr{L}_{p}\right)$ depends on $\mathscr{A}$, $\Sigma_{1}, \ldots, \Sigma_{p}$ and not just on the loop algebra $\mathscr{L}_{p}$.)

Next suppose that $0 \leq p \leq n-1$. Then $\Sigma_{p+1}$ is a $\mathbb{Z}_{m_{p+1}}$-grading of the algebra $\mathscr{L}_{p}$ which we write as

$$
\mathscr{L}_{p}=\bigoplus_{\bar{\imath} \in \mathbb{Z}_{m_{p+1}}}\left(\mathscr{L}_{p}\right)_{\bar{\imath}}
$$

We set

$$
\bar{C}\left(\mathscr{L}_{p}\right)_{\bar{l}}:=\left\{u \in \bar{C}\left(\mathscr{L}_{p}\right) \mid u \cdot\left(\mathscr{L}_{p}\right)_{\bar{\jmath}} \subset\left(\mathscr{L}_{p}\right)_{\bar{l}+\bar{\jmath}} \text { for all } \bar{j} \in \mathbb{Z}_{m_{p+1}}\right\}
$$

for $\bar{l} \in \mathbb{Z}_{m_{p+1}}$. We denote the collection $\left\{\bar{C}\left(\mathscr{L}_{p}\right)_{\bar{l}}\right\}_{\bar{i} \in \mathbb{Z}_{m_{p+1}}}$ by $\bar{C}\left(\Sigma_{p+1}\right)$. We will see in Lemma 6.1(ii) below that $\bar{C}\left(\Sigma_{p+1}\right)$ is a $\mathbb{Z}_{m_{p+1}}$-grading of $\bar{C}\left(\mathscr{L}_{p}\right)$.

Finally for $0 \leq p \leq n$ we define $\gamma_{p}: \bar{C}\left(\mathscr{L}_{p}\right) \rightarrow C\left(\mathscr{L}_{p}\right)$ by

$$
\gamma_{p}(u)(x)=u \cdot x
$$

for $u \in \bar{C}\left(\mathscr{L}_{p}\right), x \in \mathscr{L}_{p}$, in which case $\gamma_{p}$ is an algebra homomorphism. Note in particular that $\bar{C}\left(\mathscr{L}_{0}\right)=C(\mathscr{A})$ and $\gamma_{0}$ is the identity map.

Lemma 6.1. Suppose that $\mathscr{L}=L\left(\mathscr{A}, \Sigma_{1}, \ldots, \Sigma_{n}\right)$, where $\mathscr{A}$ is a pfgc algebra.

(i) If $0 \leq p \leq n$ then $\gamma_{p}: \bar{C}\left(\mathscr{L}_{p}\right) \rightarrow C\left(\mathscr{L}_{p}\right)$ is an isomorphism of $k$-algebras.

(ii) If $0 \leq p \leq n-1$ then $\bar{C}\left(\Sigma_{p+1}\right)$ is a $\mathbb{Z}_{m_{p+1}}$-grading of the algebra $\bar{C}\left(\mathscr{L}_{p}\right)$ and the map $\gamma_{p}$ is an isomorphism of graded algebras.

(iii) If $0 \leq p \leq n-1$ then

$$
\bar{C}\left(\mathscr{L}_{p+1}\right)=L\left(\bar{C}\left(\mathscr{L}_{p}\right), \bar{C}\left(\Sigma_{p+1}\right), z_{p+1}\right) .
$$


Proof. (i) We first show that $\gamma_{p}$ is injective for $0 \leq p \leq n$. To see this, suppose that $u \in \operatorname{ker}\left(\gamma_{p}\right)$. Then $u \cdot x=0$ for all $x \in \mathscr{L}_{p}$, and so (since $\mathscr{L}_{p}$ spans $\mathscr{A} \otimes_{k} S^{\otimes p}$ over $S^{\otimes p}$ by Lemma 5.7) we have $u \cdot x=0$ for all $x \in A_{\otimes_{k}} S^{\otimes p}$. This implies that $u=0$.

Next we prove the bijectively of $\gamma_{p}$ for $0 \leq p \leq n$ by induction on $p$. This is clear if $p=0$ since $\gamma_{0}$ is the identity map. So we suppose that $0 \leq p \leq n-1$ and that $\gamma_{p}$ is bijective. It is clear from this bijectivity and from the definitions of $\bar{C}\left(\mathscr{L}_{p+1}\right)$ and $C\left(\mathscr{L}_{p+1}\right)$ (see (6-1) and (4-2)) that

$$
\gamma_{p}\left(\bar{C}\left(\mathscr{L}_{p+1}\right)_{\bar{l}}\right)=C\left(\mathscr{L}_{p+1}\right)_{\bar{l}}
$$

for $\bar{\imath} \in \mathbb{Z}_{m_{p+1}}$. Hence $\bar{C}\left(\Sigma_{p+1}\right)$ is a grading of the algebra $\bar{C}\left(\mathscr{L}_{p}\right)$ and $\gamma_{p}: \bar{C}\left(\mathscr{L}_{p}\right) \rightarrow$ $C\left(\mathscr{L}_{p}\right)$ is a graded isomorphism. So $\gamma_{p}$ induces an algebra isomorphism

$$
L\left(\gamma_{p}\right): L\left(\bar{C}\left(\mathscr{L}_{p}\right), \bar{C}\left(\Sigma_{p+1}\right), z_{p+1}\right) \rightarrow L\left(C\left(\mathscr{L}_{p}\right), C\left(\Sigma_{p+1}\right), z_{p+1}\right) .
$$

Consequently we have the composite algebra isomorphism (6-3)

$$
L\left(\bar{C}\left(\mathscr{L}_{p}\right), \bar{C}\left(\Sigma_{p+1}\right), z_{p+1}\right) \stackrel{L\left(\gamma_{p}\right)}{\longrightarrow} L\left(C\left(\mathscr{L}_{p}\right), C\left(\Sigma_{p+1}\right), z_{p+1}\right) \stackrel{\psi_{\mathscr{L}_{p}, \Sigma_{p+1}}}{\longrightarrow} C\left(\mathscr{L}_{p+1}\right),
$$

where $\psi_{\mathscr{L}_{p}, \Sigma_{p+1}}$ is the isomorphism of Proposition 4.11. (Note that the proposition can be applied since $\mathscr{L}_{p}$ is a pfgc algebra by, Theorem 5.5(iii).) But

$$
L\left(\bar{C}\left(\mathscr{L}_{p}\right), \bar{C}\left(\Sigma_{p+1}\right), z_{p+1}\right) \subset \bar{C}\left(\mathscr{L}_{p+1}\right)
$$

and one easily checks that the restriction

$$
\left.\gamma_{p+1}\right|_{L\left(\bar{C}\left(\mathscr{L}_{p}\right), \bar{C}\left(\Sigma_{p+1}\right), z_{p+1}\right)}: L\left(\bar{C}\left(\mathscr{L}_{p}\right), \bar{C}\left(\Sigma_{p+1}\right), z_{p+1}\right) \rightarrow C\left(\mathscr{L}_{p+1}\right)
$$

equals the composite map (6-3). Hence the restriction (6-4) of $\gamma_{p+1}$ is bijective. Thus, since $\gamma_{p+1}$ itself is injective, it follows that

$$
L\left(\bar{C}\left(\mathscr{L}_{p}\right), \bar{C}\left(\Sigma_{p+1}\right), z_{p+1}\right)=\bar{C}\left(\mathscr{L}_{p+1}\right)
$$

and $\gamma_{p+1}$ is bijective. So we have proved (i).

(ii) and (iii): These were proved in the argument for (i).

Since $\mathscr{L}=\mathscr{L}_{n}$, we write $\bar{C}(\mathscr{L})=\bar{C}\left(\mathscr{L}_{n}\right)$ and so

$$
\bar{C}(\mathscr{L}):=\left\{u \in C(\mathscr{A}) \otimes_{k} S^{\otimes n} \mid u \cdot \mathscr{L} \subset \mathscr{L}\right\} .
$$

Then $\bar{C}(\mathscr{L})$ is a unital subalgebra of $C(\mathscr{A}) \otimes_{k} S^{\otimes n}$, and $\mathscr{A} \otimes_{k} S^{\otimes n}$ is a $\bar{C}(\mathscr{L})$-module. We also write $\gamma_{\mathscr{L}}=\gamma_{n}$. Thus $\gamma_{\mathscr{L}}: \bar{C}(\mathscr{L}) \rightarrow C(\mathscr{L})$ is the $k$-algebra homomorphism (said to be canonical) defined by

$$
\gamma_{\mathscr{L}}(u)(x)=u \cdot x
$$

for $u \in \bar{C}(\mathscr{L}), x \in \mathscr{L}$. 
Using Lemma 6.1 we can now give an explicit description of the centroid of an $n$-step loop algebra as an $n$-step loop algebra.

Theorem 6.2. Suppose that $\mathscr{L}=L\left(\mathscr{A}, \Sigma_{1}, \ldots, \Sigma_{n}\right)$ is an $n$-step loop algebra based on a pfgc algebra $\mathscr{A}$. Then the canonical map $\gamma_{\mathscr{L}}: \bar{C}(\mathscr{L}) \rightarrow C(\mathscr{L})$ is an algebra isomorphism and we have

$$
\bar{C}(\mathscr{L})=L\left(C(\mathscr{A}), \bar{C}\left(\Sigma_{1}\right), \ldots, \bar{C}\left(\Sigma_{n}\right)\right) .
$$

Proof. $\gamma_{\mathscr{L}}$ is an isomorphism by Lemma 6.1(i). Moreover (6-5) follows by repeated application of (6-2).

Corollary 6.3. Suppose that $\mathscr{L}$ is an $n$-step loop algebra based on a pfgc algebra A. Then

(i) $C(\mathscr{A})$ and $C(\mathscr{L})$ are nonzero unital commutative associative algebras over $k$.

(ii) If $C(\mathscr{A})$ is an integral domain, then $C(\mathscr{L})$ is an integral domain.

(iii) If $C(\mathscr{A})$ is finitely generated as an algebra over $k$, then $C(\mathscr{L})$ is finitely generated as an algebra over $k$ and $\operatorname{Dim} C(\mathscr{L})=\operatorname{Dim} C(\mathscr{A})+n$.

Proof. (i) Since $\mathscr{A}$ is pfgc, we know that $\mathscr{L}$ is pfgc by Theorem 5.5(iii). Hence $C(\mathscr{A})$ and $C(\mathscr{L})$ are nonzero unital commutative associative algebras by Proposition 2.11(i).

(ii) and (iii): We know by Theorem 6.2 that $C(\mathscr{L})$ is isomorphic to an $n$-step loop algebra based on $C(\mathscr{A})$. Thus (ii) and (iii) follow from Theorem 5.5 (viii) and (x) respectively.

If $\mathscr{A}$ is a finite dimensional central simple algebra over $k$, then $C(\mathscr{A})=k$ and $\mathscr{A}$ is a pfgc algebra. Hence we have the following consequence of Corollary 6.3:

Corollary 6.4. Suppose that $\mathscr{L}$ is an n-step loop algebra based on a finite dimensional central simple algebra $\mathscr{A}$ over $k$. Then $C(\mathscr{L})$ is an integral domain, $C(\mathscr{L})$ is finitely generated as an algebra over $k$, and $\operatorname{Dim} C(\mathscr{L})=n$. Consequently, if $\mathscr{L}^{\prime}$ is an $n^{\prime}$-step loop algebra based on a finite dimensional central simple algebra $\mathscr{A}^{\prime}$ over $k$, then

$$
\mathscr{L} \simeq_{k} \mathscr{L}^{\prime} \Longrightarrow n=n^{\prime} .
$$

Remark 6.5. Suppose that $\mathscr{L}$ is an $n$-step loop algebra based on a finite dimensional central simple algebra $\mathscr{A}$ over $k$. Then $C(\mathscr{A}) \otimes_{k} S^{\otimes n}=k \otimes_{k} S^{\otimes n}=S^{\otimes n}$ and so

$$
C(\mathscr{L}){\stackrel{\gamma_{\mathscr{L}}}{\simeq}}_{k} \bar{C}(\mathscr{L})=\left\{u \in S^{\otimes n}: u \cdot \mathscr{L} \subset \mathscr{L}\right\} .
$$

This fact can be used to explicitly compute $C(\mathscr{L})$ in examples. 
Corollary 6.6. Suppose that $\mathscr{L}=M\left(\mathscr{A}, \sigma_{1}, \ldots, \sigma_{n}\right)$ is a multiloop algebra based on a finite dimensional central simple algebra A over $k$, where $\sigma_{1}, \ldots, \sigma_{n}$ are commuting finite order automorphisms of $A_{A}$ with periods $m_{1}, \ldots, m_{n}$ respectively. Then

$$
\bar{C}(\mathscr{L})=k\left[\left(z_{1}^{m_{1}}\right)^{ \pm 1}, \ldots,\left(z_{n}^{m_{n}}\right)^{ \pm 1}\right],
$$

and so $C(\mathscr{L})$ is isomorphic to the algebra of Laurent polynomials in n-variables over $k$.

Proof. Recall (using the notation of Example 5.4) that

$$
\mathscr{L}=\bigoplus_{\left(i_{1}, \ldots, i_{n}\right) \in \mathbb{Z}^{n}} \mathscr{A}_{\bar{l}_{1}, \ldots, \bar{i}_{n}} \otimes_{k} z_{1}^{i_{1}} \ldots z_{n}^{i_{n}}
$$

and so the inclusion " $\supset$ " in (6-6) is clear. For the inclusion " $\subset$ ", let $u \in \bar{C}(\mathscr{L})$. Now $S^{\otimes n}$ is naturally $\mathbb{Z}^{n}$-graded and it is clear that $\bar{C}(\mathscr{L})$ is a graded subalgebra. Hence we can assume that $u=z_{1}^{j_{1}} \ldots z_{n}^{j_{n}}$, where $\left(j_{1}, \ldots, j_{n}\right) \in \mathbb{Z}^{n}$. But then $\mathscr{A}_{\bar{l}_{1}, \ldots, \bar{l}_{n}} \subset \mathscr{A}_{\bar{l}_{1}+\bar{\jmath}_{1}, \ldots, \bar{l}_{n}+\bar{\jmath}_{n}}$ for all $\left(i_{1}, \ldots, i_{n}\right) \in \mathbb{Z}^{n}$ and so $\left(\bar{\jmath}_{1}, \ldots, \bar{j}_{n}\right)=(\overline{0}, \ldots, \overline{0})$.

\section{Untwisting iterated loop algebras}

In this section we show that any $n$-step loop algebra based on a pfgc algebra can be untwisted by an extension of the centroid of $\mathscr{L}$ that is free of finite rank.

Suppose again throughout the section that $\mathscr{L}=L\left(\mathscr{A}, \Sigma_{1}, \ldots, \Sigma_{n}\right)$ is an $n$-step loop algebra based on an algebra $\mathscr{A}$ over $k$. We use the notation of the previous section.

It will be convenient to work with $\bar{C}(\mathscr{L})$ rather than $C(\mathscr{L})$ (although one could use $\gamma_{\mathscr{L}}$ to identify these algebras using Theorem 6.2 and avoid this distinction). Note that $\bar{C}(\mathscr{L})$ is a subalgebra of $C(\mathscr{A}) \otimes_{k} S^{\otimes n}$, and so $C(\mathscr{A}) \otimes_{k} S^{\otimes n} / \bar{C}(\mathscr{L})$ is a ring extension. This is the extension that we use to untwist $\mathscr{L}$.

We define

$$
\omega_{\mathscr{L}}: \mathscr{L} \otimes_{\bar{C}(\mathscr{L})}\left(C(\mathscr{A}) \otimes_{k} S^{\otimes n}\right) \rightarrow \mathscr{A} \otimes_{k} S^{\otimes n}
$$

by

$$
\omega_{\mathscr{L}}(x \otimes u)=u \cdot x
$$

for $x \in \mathscr{L}$ and $u \in C(\mathscr{A}) \otimes_{k} S^{\otimes n}$. One easily checks that $\omega_{\mathscr{L}}$ is a well-defined $C(\mathscr{A}) \otimes_{k} S^{\otimes n}$-algebra homomorphism which we call canonical.

Our untwisting theorem is the following:

Theorem 7.1. Suppose that $\mathscr{L}=L\left(\mathscr{A}, \Sigma_{1}, \ldots, \Sigma_{n}\right)$ is an $n$-step loop algebra based on a pfgc algebra $\mathscr{A}$, where $\Sigma_{p}$ has modulus $m_{p}$ for $1 \leq p \leq n$. Then 
(i) $C(\mathscr{A}) \otimes_{k} S^{\otimes n}$ is a free $\bar{C}(\mathscr{L})$-module of rank $m_{1} \ldots m_{n}$ with basis

$$
\left\{1_{C(\mathscr{A})} \otimes z^{\mathbf{i}} \mid \mathbf{i} \in I_{n}\right\} .
$$

(ii) The canonical map $\omega \mathscr{L}$ is an isomorphism and so

$$
\mathscr{L} \otimes_{\bar{C}(\mathscr{L})}\left(C(\mathscr{A}) \otimes_{k} S^{\otimes n}\right) \simeq C(\mathscr{A}) \otimes_{k} S^{\otimes n} \mathscr{A} \otimes_{k} S^{\otimes n} .
$$

Proof. (i) Since $\bar{C}(\mathscr{L})$ is an $n$-step loop algebra based on $C(\mathscr{A})$ by Theorem 6.2, statement (i) follows from Corollary 5.8.

(ii) First $\mathscr{L}$ spans $\mathscr{A} \otimes_{k} S^{\otimes n}$ over $S^{\otimes n}$ by Lemma 5.7, and so $\mathscr{L}$ spans $\mathscr{A} \otimes_{k} S^{\otimes n}$ over $C(\mathscr{A}) \otimes S^{\otimes n}$. Thus $\omega_{\mathscr{L}}$ is surjective.

To show that $\omega_{\mathscr{L}}$ is injective, let $x \in \operatorname{ker}\left(\omega_{\mathscr{L}}\right)$. Then, in particular, $x$ is an element of $\mathscr{L} \otimes_{\bar{C}(\mathscr{L})}\left(C(\mathscr{A}) \otimes_{k} S^{\otimes n}\right)$. Now since $\bar{C}(\mathscr{L})$ is an $n$-step loop algebra based on $C(\mathscr{A})$, it follows from Lemma 5.7 that every element of $C(\mathscr{A}) \otimes_{k} S^{\otimes n}$ can be written as a sum of elements of the form $z^{\mathbf{i}} \cdot u$, where $\mathbf{i} \in I_{n}$ and $u \in \bar{C}(\mathscr{L})$. But $z^{\mathbf{i}} \cdot u=u \cdot\left(1_{C(\mathscr{A})} \otimes z^{\mathbf{i}}\right)$. Thus from the balanced property in the tensor product $\mathscr{L} \otimes_{\bar{C}(\mathscr{L})}\left(C(\mathscr{A}) \otimes_{k} S^{\otimes n}\right)$, it follows that $x$ can be written in the form

$$
x=\sum_{\mathbf{i} \in I_{n}} x_{\mathbf{i}} \otimes\left(1_{C(\mathscr{A})} \otimes z^{\mathbf{i}}\right),
$$

where $x_{\mathbf{i}} \in \mathscr{L}$ for all $\mathbf{i}$. Applying $\omega_{\mathscr{L}}$ to this expression yields $\sum_{\mathbf{i} \in I_{n}} z^{\mathbf{i}} \cdot x_{\mathbf{i}}=0$, and so $x_{\mathbf{i}}=0$ for all $\mathbf{i} \in I_{n}$ by Lemma 5.7. Thus $x=0$ and $\omega \mathscr{L}$ is injective.

Remark 7.2. Suppose that $\mathscr{L}$ is an $n$-step loop algebra based on a pfgc algebra $\mathscr{A}$.

(i) We can use the canonical isomorphism $\gamma_{\mathscr{L}}: \bar{C}(\mathscr{L}) \rightarrow C(\mathscr{L})$ of Theorem 6.2 to identify the algebras $\bar{C}(\mathscr{L})$ and $C(\mathscr{L})$. This identification is compatible with the actions of these algebras on $\mathscr{L}$ and it gives $C(\mathscr{A}) \otimes_{k} S^{\otimes n}$ the structure of a $C(\mathscr{L})$-module. Then (7-1) can be restated as

$$
\mathscr{L} \otimes_{C(\mathscr{L})}\left(C(\mathscr{A}) \otimes_{k} S^{\otimes n}\right) \simeq C(\mathscr{A}) \otimes_{k} S^{\otimes n} \mathscr{A} \otimes_{k} S^{\otimes n} .
$$

Since $\mathscr{A} \otimes_{k} S^{\otimes n}$ is the untwisted $n$-step loop algebra based on $\mathscr{A}$, Theorem 7.1 tells us that $\mathscr{L}$ is untwisted by a free base ring extension of rank $m_{1} \ldots m_{n}$ of the centroid of $\mathscr{L}$.

(ii) Also observe that the algebras $\mathscr{A} \otimes_{k} S^{\otimes n}$ and $\mathscr{A} \otimes_{C(\mathscr{A})}\left(C(\mathscr{A}) \otimes_{k} S^{\otimes n}\right)$ are canonically isomorphic as $C(\mathscr{A}) \otimes_{k} S^{\otimes n}$-algebras. Thus the isomorphism (7-1') can be further restated as

$$
\mathscr{L} \otimes_{C(\mathscr{L})}\left(C(\mathscr{A}) \otimes_{k} S^{\otimes n}\right) \simeq C(\mathscr{A}) \otimes_{k} S^{\otimes n} \mathscr{A} \otimes_{C(\mathscr{A})}\left(C(\mathscr{A}) \otimes_{k} S^{\otimes n}\right) .
$$

Theorem 7.1 can be used to compare properties of an iterated loop algebra as a module or algebra over its centroid with corresponding properties of the base algebra over its centroid. We now indicate an example of this sort of argument. 
Corollary 7.3. Let $\mathscr{L}$ be an $n$-step loop algebra based on a pfgc algebra $A$. If $\mathscr{A}$ is a projective $C(\mathscr{A})$-module then $\mathscr{L}$ is a finitely generated projective $C(\mathscr{L})$-module

Proof. As in Remark 7.2, we identify $\bar{C}(\mathscr{L})$ and $C(\mathscr{L})$ using $\gamma_{\mathscr{L}}$. By axiom P2 of pfgc algebras and the present assumption, $\mathscr{A}$ is a finitely generated projective $C(\mathscr{A})$-module. Hence $\mathscr{A} \otimes_{C(\mathscr{A})}\left(C(\mathscr{A}) \otimes_{k} S^{\otimes n}\right)$ is a finitely generated projective $C(\mathscr{A}) \otimes_{k} S^{\otimes n}$-module. Thus by $\left(7-1^{\prime \prime}\right), \mathscr{L} \otimes_{C(\mathscr{L})}\left(C(\mathscr{A}) \otimes_{k} S^{\otimes n}\right)$ is a finitely generated projective $C(\mathscr{A}) \otimes_{k} S^{\otimes n}$-module. But the extension $C(\mathscr{A}) \otimes_{k} S^{\otimes n} / C(\mathscr{L})$ is free of finite rank by Theorem 7.1(i), and so it is faithfully flat. The result now follows from [Bourbaki 1972, Ch. I, § 3.6, Prop. 12]

In the same spirit, we now describe an application of Theorem 7.1 for associative algebras. For this purpose we first recall some definitions and basic facts about Azumaya algebras. A unital associative algebra $\mathscr{D}$ over a ring $B$ is called an Azumaya algebra over $B$ if $\mathscr{D}$ is central and separable over $B$ (see for example [Knus and Ojanguren 1974, §5]). If $\mathscr{D}$ is an Azumaya algebra over $B$, then $\mathscr{D}$ is a finitely generated projective $B$-module [Knus and Ojanguren 1974, Théorème 5.1], and so $\mathscr{D}_{\mathfrak{m}}$ is a free $B_{\mathfrak{m}}$-module of finite rank $r_{\mathfrak{m}}$ for each maximal ideal $\mathfrak{m}$ of $B$. $\mathscr{D}$ is said to have constant rank $r$ over $B$ if $r_{\mathfrak{m}}=r$ for all such $\mathfrak{m}$ [Bourbaki 1972, $\S$ II.5.3]. It is known that if $\mathscr{D}$ is a unital associative algebra over a $\operatorname{ring} B$ and $\ell$ is a positive integer then

$\mathscr{D}$ is an Azumaya algebra of constant rank $\ell^{2}$ over $B$ if and only if there exists a faithfully flat extension $B^{\prime} / B$ of rings so that $\mathscr{D} \otimes_{B}$ $B^{\prime}$ is isomorphic as a $B^{\prime}$-algebra to the algebra $M_{\ell}\left(B^{\prime}\right)$ of $\ell \times \ell$ matrices over $B^{\prime}$.

In that case we will say that $\mathscr{D}$ is split by the extension $B^{\prime} / B$. Indeed the implication " $\Rightarrow$ " of (7-2) is Corollary 6.7 of [Knus and Ojanguren 1974]. For the converse, the algebra $M_{\ell}\left(B^{\prime}\right)$ is an Azumaya algebra of constant rank $\ell^{2}$ over $B^{\prime}$, and hence $\mathscr{D}$ is an Azumaya algebra of constant rank $\ell^{2}$ over $B$ since the extension $B^{\prime} / B$ is faithfully flat (see Lemma 5.1.9(1) in [Knus 1991] and Exercise 8 in [Bourbaki 1972, § II.5]).

Corollary 7.4. Suppose that $\mathscr{L}$ is an $n$-step loop algebra based on the matrix algebra $M_{\ell}(k)$ over $k$. Then $\mathscr{L}$ is a prime Azumaya algebra of finite rank $\ell^{2}$ over its centroid $C(\mathscr{L})$ which is split by the extension $S^{\otimes n} / C(\mathscr{L})$.

Proof. Let $\mathscr{A}=M_{\ell}(k)$. Then $\mathscr{A}$ is a prime unital associative algebra over $k$ and hence so is $\mathscr{L}$ (by Theorem 5.5). Also $C(\mathscr{A})=k$ and so $C(\mathscr{A}) \otimes_{k} S^{\otimes n}=S^{\otimes n}$ as in Remark 6.5. Thus by $\left(7-1^{\prime}\right)$ we have

$$
\mathscr{L} \otimes_{C(\mathscr{L})} S^{\otimes n} \simeq_{S^{\otimes n}} M_{\ell}(k) \otimes_{k} S^{\otimes n} \simeq_{S^{\otimes n}} M_{\ell}\left(S^{\otimes n}\right) .
$$


Our conclusion now follows from (7-2), since the extension $S^{\otimes n} / C(\mathscr{L})$ is faithfully flat.

\section{Permanence of type}

There is a classical notion of type for simple pfgc Lie algebras in characteristic zero (see Example 8.1 below). This notion can easily be extended using the central closure to include prime pfgc Lie algebras in characteristic 0 . It will be a consequence of the results in this section that type is preserved under the loop construction (that is type is permanent).

An analogous notion of type can be defined for many other important classes of prime pfgc algebras besides Lie algebras in characteristic 0. Moreover, since algebras in these classes arise naturally as coordinate algebras in the study of Lie algebras, and in particular in the study of extended affine Lie algebras, it is desirable to include these classes in our discussion of type. This generality requires almost no extra effort once the appropriate definitions are made. That being said, the reader can safely choose to assume throughout this section that the base algebras, and hence their loop algebras, are Lie algebras in characteristic 0 .

We begin by recalling the classical notion of type for simple pfgc Lie algebras in characteristic 0 .

Example 8.1. Suppose that $k$ has characteristic 0 . Let $\mathscr{A}$ be a simple pfgc Lie algebra over $k$ (or equivalently let $\mathscr{A}$ be a simple Lie algebra over $k$ that is finitely generated as a module over its centroid). Then, since $\mathscr{A}$ is simple, it is easily checked that $C(\mathscr{A})$ is a field. Hence, if we let $K$ be an algebraic closure of $C(\mathscr{A})$, the algebra $\mathscr{A}_{C(\mathscr{A})} K$ is a finite dimensional simple Lie algebra over $K$ by Lemma 2.4. The type of $\mathscr{A}$ is defined in [Jacobson 1962, Ch. X, §3] to coincide with the type of the root system of the $K$-algebra $\mathscr{A}_{C(\mathscr{A})} K$ relative to any Cartan subalgebra.

In order to extend this notion to other classes of algebras, we need to introduce some terminology.

Definition 8.2. Recall that a variety over $k$ is a class $\mathbb{V}_{k}$ of algebras over $k$ that is defined by a set of identities in the free nonassociative algebra $k_{\mathrm{na}}\left[x_{1}, x_{2}, \ldots\right]$ in countably many symbols [Zhevlakov et al. 1982, § 1.2]. A variety $\mathbb{V}_{k}$ over $k$ is said to be homogeneous if the ideal in $k_{\mathrm{na}}\left[x_{1}, x_{2}, \ldots\right]$ consisting of all identities satisfied by all algebras in $\mathbb{V}_{k}$ is homogeneous. Algebras in a variety $\mathbb{V}_{k}$ over $k$ will be simply called $\mathbb{V}_{k}$-algebras.

A very familiar example is the variety $\mathbb{V}_{k}$ of Lie algebras over $k$ which is defined by the identities $x_{1} x_{1}$ and $\left(x_{1} x_{2}\right) x_{3}+\left(x_{2} x_{3}\right) x_{1}+\left(x_{3} x_{1}\right) x_{2}$. In that case $\mathbb{V}_{k}$ is homogeneous [Zhevlakov et al. 1982, § 1.4], and a $\mathbb{V}_{k}$-algebra is just a Lie algebra over $k$. 
Remark 8.3. Suppose that $\mathbb{V}_{k}$ is a variety over $k$. Suppose that $B$ is a unital associative commutative $k$-algebra. A $\mathbb{V}_{k}$-algebra over $B$ will mean a $B$-algebra $\mathscr{A}$ with the property that $\mathscr{A}$, when regarded as an algebra over $k$, is in $\mathbb{V}_{k}$. In other words, a $\mathbb{V}_{k}$-algebra over $B$ is a $B$-algebra that satisfies the identities (which are identities with coefficients from our fixed base field $k$ ) that define $\mathbb{V}_{k}$.

The homogeneity assumption is important for our purposes since homogeneous varieties are closed under base ring extension.

Lemma 8.4. Suppose that $\mathbb{V}_{k}$ is a homogeneous variety over $k$ and $B \rightarrow B^{\prime}$ is a homomorphism of unital commutative associative $k$-algebras. If $\mathscr{A}$ is a $\mathbb{V}_{k}$-algebra over $B$ then $A \otimes_{B} B^{\prime}$ is a $\mathbb{V}_{k}$-algebra over $B^{\prime}$.

Proof. This follows the proof of Theorem 6 in [Zhevlakov et al. 1982, § 1.4].

Corollary 8.5. Suppose that $\mathbb{V}_{k}$ is a homogeneous variety over $k$. If $\mathscr{L}$ is an $n$-step loop algebra based on a $\mathbb{V}_{k}$-algebra $\mathscr{A}$, then $\mathscr{L}$ is a $\mathbb{V}_{k}$-algebra.

Proof. By Lemma $8.4, \mathscr{A} \otimes_{k} S^{\otimes n}$ is a $\mathbb{V}_{k}$-algebra. Hence so is its subalgebra $\mathscr{L}$.

We will be interested in homogeneous varieties $\mathbb{V}_{k}$ that satisfy the following axiom:

(S) If $K / k$ is a field extension and $\mathscr{A}$ is a finite dimensional semiprime $\mathbb{V}_{k}$-algebra over $K$ then $\mathscr{A}$ is a direct sum of simple algebras over $K$.

Example 8.6. In each of the following cases, the variety $\mathbb{V}_{k}$ is homogeneous and satisfies axiom $(\mathrm{S})$ :

(a) $\operatorname{char}(k)=0, \mathbb{V}_{k}$ is the variety of Lie algebras.

(b) $\mathbb{V}_{k}$ is the variety of associative algebras.

(c) $\mathbb{V}_{k}$ is the variety of commutative associative algebras.

(d) $\mathbb{V}_{k}$ is the variety of alternative algebras.

(e) $\operatorname{char}(k) \neq 2, \mathbb{V}_{k}$ is the variety of Jordan algebras.

Indeed the fact that these varieties are homogeneous is proved in [Zhevlakov et al. $1982, \S 1.4]$. Moreover axiom (S) follows from the structure theory for the variety $\mathbb{V}_{k}$ in each case. For example, in case (a), suppose that $K / k$ is a field extension and $\mathscr{A}$ is a finite dimensional semiprime Lie algebra over $K$. If the radical $\mathscr{R}$ of $\mathscr{A}$ is nonzero, then the last nonzero term in the derived series for $\mathscr{R}$ has trivial multiplication, contrary to the assumption that $\mathscr{A}$ is semiprime. So $\mathscr{R}=0$ and hence $\mathscr{A}$ is the direct sum of simple algebras [Jacobson 1962, § III.4]. Similarly we can use (for example) [Zhevlakov et al. 1982, §12.2, Theorem 3] in cases (b), (c) and (d) and [Jacobson 1968, § V.2, Lemma 2 and $\S$ V.5, Corollary 2] in case (e) to verify axiom $(\mathrm{S})$. 
The reason for our interest in Axiom (S) is that it allows us to prove the following proposition.

Proposition 8.7. Let $\mathbb{V}_{k}$ be a homogeneous variety over $k$ that satisfies axiom (S). Suppose that $\mathscr{A}$ is a prime pfgc $\mathbb{V}_{k}$-algebra over $k$ and let $\widetilde{C(\mathscr{A})}$ be the quotient field of $C(\mathscr{A})$. Then the central closure $\widetilde{A}:=\mathscr{A} \otimes_{C(\mathscr{A})} \widetilde{C(\mathscr{A})}$ of $\mathscr{A}$ is a finite dimensional central simple $\mathbb{V}_{k}$-algebra over $\widetilde{C(\mathscr{A})}$.

Proof. By Proposition 3.5, $\widetilde{A}$ is a prime pfgc algebra over $k$ and hence also over $\widetilde{C(\mathscr{A})}$ (see Remark 2.10 and Lemma 3.2). Also, by Lemma $8.4, \widetilde{A}$ is a $\mathbb{V}_{k}$-algebra. Hence, by axiom $(\mathrm{S}), \widetilde{A}$ is the direct sum of simple algebras over $\widetilde{C(\mathscr{A})}$. Since $\widetilde{A}$ is prime, there is only one summand in this sum. Thus $\tilde{\mathscr{A}}$ is a simple algebra over $\widetilde{C(\mathscr{A})}$. Finally, by Proposition $3.5, \widetilde{A}$ is central and finite dimensional over $\widetilde{C(\mathscr{A})}$.

Remark 8.8. Suppose that $\mathscr{A}$ is as in Proposition 8.7. Then in the terminology of [Polikarpov and Shestakov 1990, §1], Proposition 8.7 says that $\mathscr{A}$ is a central order in the finite dimensional central simple algebra $\tilde{A}$.

We will also need a set $\mathbb{X}_{k}$ of algebras over $k$ that play the role of the split simple Lie algebras over $k$.

Definition 8.9. Suppose that $\mathbb{V}_{k}$ is a homogeneous variety over $k$. A set of archetypes for $\mathbb{V}_{k}$ is a set $\mathbb{X}_{k}$ of finite dimensional central simple $\mathbb{V}_{k}$-algebras over $k$ such that the following axioms hold:

(A1) If $K / k$ is an algebraically closed field extension and $\mathscr{A}$ is a finite dimensional central simple $\mathbb{V}_{k}$-algebra over $K$ then there exists $\mathscr{X} \in \mathbb{X}_{k}$ so that $\mathscr{A} \simeq_{K} \mathscr{X} \otimes_{k} K$.

(A2) If $K / k$ is a field extension and $\mathscr{X}, \mathscr{X}^{\prime} \in \mathbb{X}_{k}$ then

$$
\mathscr{L} \otimes_{k} K \simeq_{K} \mathscr{X}^{\prime} \otimes_{k} K \Longrightarrow \mathscr{X}=\mathscr{X}^{\prime}
$$

In particular, the elements of $\mathbb{X}_{k}$ are pairwise nonisomorphic over $k$.

Example 8.10. In each of the cases (a)-(e) in Example 8.6 there is a natural choice for a set $\mathbb{X}_{k}$ of archetypes:

(a) $\operatorname{char}(k)=0, \mathbb{V}_{k}$ is the variety of Lie algebras and $\mathbb{X}_{k}=\left\{\mathscr{X}_{\Pi}\right\}$, where $\Pi$ runs through all connected Dynkin diagrams (up to isomorphism) and $\mathscr{X}_{\Pi}$ denotes the split simple Lie algebra with Dynkin diagram П [Jacobson 1962, § VII.4].

(b) $\mathbb{V}_{k}$ is the variety of associative algebras and $\mathbb{X}_{k}=\left\{M_{\ell}(k) \mid \ell \geq 1\right\}$, where $M_{\ell}(k)$ is the algebra of $\ell \times \ell$-matrices over $k$.

(c) $\mathbb{V}_{k}$ is the variety of commutative associative algebras and $\mathbb{X}_{k}=\{k\}$.

(d) $\mathbb{V}_{k}$ is the variety of alternative algebras and $\mathbb{X}_{k}=\left\{M_{\ell}(k) \mid \ell \geq 1\right\} \cup\{\mathcal{O}\}$, where O denotes the split Cayley-Dickson (octonion) algebra [Zhevlakov et al. 1982, $\S 2.4]$. 
(e) $\operatorname{char}(k) \neq 2, \mathbb{V}_{k}$ is the variety of Jordan algebras and $\mathbb{X}_{k}$ is the set consisting of the following algebras: $k$; the Jordan algebra constructed from a nondegenerate symmetric bilinear form with matrix $\operatorname{diag}(1,-1, \ldots, 1,-1)$ on a $2 \ell$-dimensional space over $k, \ell \geq 1$; the Jordan algebra constructed from a nondegenerate symmetric bilinear form with matrix $\operatorname{diag}(1,-1, \ldots, 1,-1,1)$ on a $2 \ell+1$-dimensional space over $k, \ell \geq 1$; the algebra of $\ell \times \ell$ hermitian matrices with coordinates from the split composition algebras of dimension 1 , 2 and $4, \ell \geq 3$; and the algebra of $3 \times 3$ hermitian matrices over 0 [Jacobson $1968, \S 1.4$ and 4.3].

The fact that $\mathbb{X}_{k}$ satisfies axioms (A1) and (A2) follows from the classification of finite dimensional central simple algebras over algebraically closed fields in each case. See for example [Jacobson 1962, § IV.3, Theorem 3] in case (a), [Zhevlakov et al. 1982, § 12.2, Theorem 3] in cases (b), (c) and (d), and [Jacobson 1968, § V.6, Corollary 2] in case (e).

Remark 8.11. A homogeneous variety $\mathbb{V}_{k}$ over $k$ may possess more than one set of archetypes. For example if $k=\mathbb{R}$ and $\mathbb{V}_{k}$ is the variety of Lie algebras over $k$ as in Example 8.10(a) above, then an alternate choice of a set of archetypes is the set $\mathbb{X}_{k}=\left\{\mathscr{C}_{\Pi}\right\}$, where $\Pi$ runs through all connected Dynkin diagrams (up to isomorphism) and $\mathscr{C}_{\Pi}$ denotes the compact real Lie algebra whose complexification is the simple Lie algebra with Dynkin diagram $\Pi$.

Assumption. For the rest of this section we assume that $\mathbb{V}_{k}$ is a homogeneous variety over $k$ that satisfies axiom (S), and that there exists a set $\mathbb{X}_{k}$ (which we fix) of archetypes for $\mathbb{V}_{k}$.

We can now prove the proposition that allows us to define the notion of type.

Proposition 8.12. Suppose that $\mathscr{A}$ is a prime pfgc $\mathbb{V}_{k}$-algebra over $k$. If

$$
C(\mathscr{A}) \hookrightarrow K
$$

is a unital k-algebra monomorphism of $C(\mathscr{A})$ into an algebraically closed field extension $K$ of $k$ (such a monomorphism exists since $C(\mathscr{A})$ is an integral domain), then there exists a unique $\mathscr{X} \in \mathbb{X}_{k}$ so that

$$
\mathscr{A} \otimes_{C(\mathscr{A})} K \simeq_{K} \mathscr{L} \otimes_{k} K
$$

where on the left $K$ is regarded as an algebra over $C(A)$ using the given monomorphism. Moreover, $\mathscr{X}$ is independent of the choice of the $k$-algebra monomorphism $C(\mathscr{A}) \hookrightarrow K$. 
Proof. First let $L$ be an algebraic closure of $\widetilde{C(\mathscr{A})}$. By Proposition $8.7, \widetilde{A}$ is a finite dimensional central simple $\mathbb{V}_{k}$-algebra over $\widetilde{C(\mathscr{A})}$. Therefore, by Lemma 2.4, $\widetilde{A} \otimes \widetilde{C(\mathscr{A})} L$ is a finite dimensional central simple algebra over $L$. So, by Lemma 8.4, $\widetilde{A} \otimes \frac{(\mathscr{A})}{C(\mathbb{A})} L$ is a finite dimensional central simple $\mathbb{V}_{k}$-algebra over $L$. Thus, by axiom (A1) (see Definition 8.9), there exists $\mathscr{X} \in \mathbb{X}_{k}$ so that $\widetilde{A} \otimes \widetilde{C(\mathscr{A})} L \simeq_{L} \mathscr{X} \otimes_{k} L$. Then

$$
\mathscr{A} \otimes_{C(\mathscr{A})} L \simeq_{L}\left(\mathscr{A} \otimes_{C(\mathscr{A})} \widetilde{C(\mathscr{A})}\right) \otimes_{\widetilde{C(\mathscr{A})}} L=\tilde{\mathscr{A}} \otimes_{\widetilde{C(\mathscr{A})}} L \simeq_{L} \mathscr{X} \otimes_{k} L .
$$

Now let $C(\mathscr{A}) \hookrightarrow K$ be an arbitrary unital $k$-algebra monomorphism of $C(\mathscr{A})$ into an algebraically closed extension $K$ of $k$. This extends to a unital $k$-algebra monomorphism $\widetilde{C(\mathscr{A})} \hookrightarrow K$ which in turns extends to a unital $k$-algebra monomorphism $L \hookrightarrow K$. We use this latter monomorphism to identify $L$ as a subfield of $K$. Then using (8-2) we have

$$
\mathscr{A} \otimes_{C(\mathscr{A})} K \simeq_{K}\left(\mathscr{A} \otimes_{C(\mathscr{A})} L\right) \otimes_{L} K \simeq_{K}\left(\mathscr{X} \otimes_{k} L\right) \otimes_{L} K \simeq_{K} \mathscr{X} \otimes_{k} K .
$$

This shows the existence of an element $\mathscr{L} \in \mathbb{X}_{k}$ satisfying (8-1). The uniqueness follows from Axiom (A2).

Finally if $C(\mathscr{A}) \hookrightarrow K^{\prime}$ is another unital $k$-algebra monomorphism of $C(\mathscr{A})$ into an algebraically closed extension $K^{\prime}$ of $k$, then the argument just given using (8-2) shows that $\mathscr{A} \otimes_{C(\mathscr{A})} K^{\prime} \simeq_{K^{\prime}} \mathscr{L} \otimes_{k} K^{\prime}$.

Definition 8.13. Let $\mathscr{A}$ be a prime pfgc $\mathbb{V}_{k}$-algebra over $k$. The element $\mathscr{X} \in \mathbb{X}_{k}$ described in Proposition 8.12 is called the type of $\mathscr{A}$ (relative to $\mathbb{X}_{k}$ ) and denoted by $t(\mathscr{A})$. We also sometimes refer to $t(\mathscr{A})$ as the absolute type of $\mathscr{A}$ since it is determined by extending the base ring $C(\mathscr{A})$ to an algebraically closed field.

Example 8.14. Let $\operatorname{char}(k)=0$, let $\mathbb{V}_{k}$ be the variety of Lie algebras, and let $\mathbb{X}_{k}=\left\{\mathscr{X}_{\Pi}\right\}$ be as in Example 8.10 (a). If we identify $\mathscr{X}_{\Pi}$ with the diagram $\Pi$, then Definition 8.13 assigns to each prime pfgc $\mathbb{V}_{k}$-algebra $\mathscr{A}$ a connected Dynkin diagram $t(\mathscr{A})$. (If $\mathscr{A}$ is a simple pfgc algebra, this is exactly what was done in Example 8.1.)

The following result tells us that type is an isomorphism invariant for prime pfgc algebras.

Proposition 8.15. Suppose that $\mathscr{A}$ and $\mathscr{A}^{\prime}$ are prime $p f g c \mathbb{V}_{k}$-algebras over $k$. Then

$$
\mathscr{A} \simeq_{k} \mathscr{A}^{\prime} \Longrightarrow t(\mathscr{A})=t\left(\mathscr{A}^{\prime}\right)
$$

Proof. Let $\varphi: C\left(\mathscr{A}^{\prime}\right) \hookrightarrow K$ be a unital $k$-algebra monomorphism of $C\left(\mathscr{A}^{\prime}\right)$ into an algebraically closed field extension $K$ of $k$. Denote the resulting action of $C\left(\mathscr{A}^{\prime}\right)$ on $K$ by $\left(\chi^{\prime}, \alpha\right) \mapsto \chi^{\prime} \cdot \alpha$. 
Fix a $k$-algebra isomorphism $\rho: \mathscr{A} \rightarrow \mathscr{A}^{\prime}$. Then $\rho$ induces an isomorphism $C(\rho): C(\mathscr{A}) \rightarrow C\left(\mathscr{A}^{\prime}\right)$ by Lemma 2.2. So the composite map $\varphi \circ C(\rho): C(\mathscr{A}) \rightarrow K$ is a unital $k$-algebra monomorphism which we use to view $K$ as an algebra over $C(\mathscr{A})$. The resulting action of $C(\mathscr{A})$ on $K$ is given by

$$
\chi \cdot \alpha=C(\rho)(\chi) \cdot \alpha .
$$

for $\chi \in C(\mathscr{A})$ and $\alpha \in K$.

The biadditive map $\tilde{\rho}: \mathscr{A} \times K \rightarrow \mathscr{A}^{\prime} \otimes_{C\left(\mathscr{A}^{\prime}\right)} K$ satisfying $\widetilde{\rho}:(a, \alpha) \mapsto \rho(a) \otimes \alpha$ is then $C(\mathscr{A})$-balanced. Indeed if $\chi \in C(\mathscr{A}), a \in \mathscr{A}$ and $\alpha \in K$ we have

$$
\begin{aligned}
\tilde{\rho}(\chi(a), \alpha) & =\rho(\chi(a)) \otimes \alpha=C(\rho)(\chi)(\rho(a)) \otimes \alpha \\
& =\rho(a) \otimes(C(\rho)(\chi) \cdot \alpha)=\rho(a) \otimes \chi \cdot \alpha=\tilde{\rho}(a, \chi \cdot \alpha) .
\end{aligned}
$$

Thus $\tilde{\rho}$ induces a $k$-linear map $\mathscr{A}_{C(\mathscr{A})} K \rightarrow \mathscr{A}^{\prime} \otimes_{C\left(\mathscr{A}^{\prime}\right)} K$ so that $a \otimes \alpha \mapsto \rho(a) \otimes \alpha$ for $a \in \mathscr{A}$ and $\alpha \in K$. This map is clearly a homomorphism of $K$-algebras. In a similar fashion we obtain a homomorphism of $K$-algebras $\mathscr{A}^{\prime} \otimes_{C\left(\mathscr{A}^{\prime}\right)} K \rightarrow \mathscr{A} \otimes_{C(\mathscr{A})}$ $K$ so that $a^{\prime} \otimes \alpha \mapsto \rho^{-1}\left(a^{\prime}\right) \otimes \alpha$ for $a^{\prime} \in \mathscr{A}^{\prime}$ and $\alpha \in K$. These maps are inverses of each other and so we have

$$
\mathscr{A} \otimes_{C(\mathscr{A})} K \simeq_{K} \mathscr{A}^{\prime} \otimes_{C\left(\mathscr{A}^{\prime}\right)} K .
$$

Thus, $\mathscr{L} \otimes_{k} K \simeq_{K} \mathscr{X}^{\prime} \otimes_{k} K$, where $\mathscr{L}=t(\mathscr{A})$ and $\mathscr{X}^{\prime}=t\left(\mathscr{A}^{\prime}\right)$, and so $t(\mathscr{A})=t\left(\mathscr{A}^{\prime}\right)$.

Our main result in this section is the following:

Theorem 8.16. (Permanence of type) If $\mathscr{L}$ is an $n$-step loop algebra based on a prime pfgc $\mathbb{V}_{k}$-algebra $\mathscr{A}$, then $\mathscr{L}$ is a prime pfgc $\mathbb{V}_{k}$-algebra and

$$
t(\mathscr{L})=t(\mathscr{A}) .
$$

Proof. By Theorem 5.5(iii) and (iv) and Corollary 8.5, $\mathscr{L}$ is a prime pfgc $\mathbb{V}_{k^{-}}$ algebra. So $t(\mathscr{A})$ and $t(\mathscr{L})$ are defined and it remains so show that these types are equal. For this note first that $C(\mathscr{A})$ is an integral domain by Lemma 3.3(i), and so the algebra $C(\mathscr{A}) \otimes_{k} S^{\otimes n} \simeq_{k} C(\mathscr{A})\left[z_{1}^{ \pm 1}, \ldots, z_{n}^{ \pm 1}\right]$ is an integral domain. Let $K$ be an algebraic closure of the quotient field of $C(\mathscr{A}) \otimes_{k} S^{\otimes n}$. Now by (7-1") we have the isomorphism

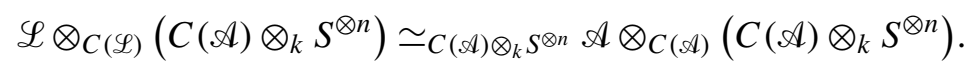

Tensoring this over $C(\mathscr{A}) \otimes_{k} S^{\otimes n}$ with $K$ yields the isomorphism

$$
\mathscr{L} \otimes_{C(\mathscr{L})} K \simeq_{K} \mathscr{A} \otimes_{C(\mathscr{A})} K .
$$

Hence we have $\mathscr{X} \otimes_{k} K \simeq_{K} \mathscr{X}^{\prime} \otimes_{k} K$, where $\mathscr{X}=t(\mathscr{A})$ and $\mathscr{X}^{\prime}=t(\mathscr{L})$, and so $\mathscr{X}=\mathscr{X}^{\prime}$. 
Since finite dimensional central simple algebras over $k$ are prime pfgc algebras, we have:

Corollary 8.17. If $\mathscr{L}$ is an $n$-step loop algebra based on a finite dimensional central simple $\mathbb{V}_{k}$-algebra $\mathscr{A}$ over $k$, then $\mathscr{L}$ is a prime pfgc $\mathbb{V}_{k}$-algebra and $t(\mathscr{L})=t(\mathscr{A})$.

Corollary 8.18. If $\mathscr{X} \in \mathbb{X}_{k}$ and $\mathscr{L}$ is an $n$-step loop algebra based on $\mathscr{X}$, then $\mathscr{L}$ is a prime pfgc $\mathbb{V}_{k}$-algebra of type $\mathscr{X}$. If further $\mathscr{X}^{\prime} \in \mathbb{X}_{k}$ and $\mathscr{L}^{\prime}$ is an $n^{\prime}$-step loop algebra based on $\mathscr{X}^{\prime}$, then

$$
\mathscr{L} \simeq_{k} \mathscr{L}^{\prime} \Longrightarrow \mathscr{X}=\mathscr{X}^{\prime} \text { and } n=n^{\prime} .
$$

Proof. The first statement follows from Corollary 8.17 since $t(\mathscr{X})=\mathscr{X}$. For the second statement suppose that $\mathscr{L} \simeq_{k} \mathscr{L}^{\prime}$. Then by Proposition $8.15, t(\mathscr{L})=t\left(\mathscr{L}^{\prime}\right)$ and so $\mathscr{X}=\mathscr{X}^{\prime}$. Finally by Corollary $6.4, n=n^{\prime}$.

Our primary focus in future work will be on the case when the base algebra is a finite dimensional split simple Lie algebra. For ease of reference we therefore record Corollary 8.18 explicitly in that case.

Corollary 8.19. Suppose that $\mathscr{A}$ is a finite dimensional split simple Lie algebra over a field $k$ of characteristic 0 , and $\mathscr{L}$ is an $n$-step loop algebra based on $\mathscr{A}$. Then $\mathscr{L}$ is a prime pfgc Lie algebra and for any unital k-algebra monomorphism $C(\mathscr{L}) \hookrightarrow K$ into an algebraically closed extension $K$ of $k$ we have

$$
\mathscr{L} \otimes_{C(\mathscr{L})} K \simeq_{K} \mathscr{A} \otimes_{k} K .
$$

Moreover, if $\mathscr{A}^{\prime}$ is a finite dimensional split simple Lie algebra and $\mathscr{L}^{\prime}$ is an $n^{\prime}$-step loop algebra based on $\mathscr{A}^{\prime}$, then

$$
\mathscr{L} \simeq_{k} \mathscr{L}^{\prime} \Longrightarrow \mathscr{A} \simeq_{k} \mathscr{A}^{\prime} \text { and } n=n^{\prime} .
$$

Proof. We apply Corollary 8.18 to the case when $\mathbb{V}_{k}$ is the variety of Lie algebras and $\mathbb{X}_{k}=\left\{\mathscr{X}_{\Pi}\right\}$ as in Example 8.10(a). Since any finite dimensional split simple Lie algebra over $k$ is isomorphic to exactly one algebra in $\mathbb{X}_{k}$ the result follows.

If $\mathscr{L}$ is an $n$-step loop algebra based on a finite dimensional split simple Lie algebra $\mathscr{A}$ (in characteristic 0 ), then (8-3) tells us that both (the isomorphism class of) the base algebra $\mathscr{A}$ and the number of steps $n$ are isomorphism invariants of $\mathscr{L}$. This answers a natural question that began the research described in this paper. We have now seen in Corollary 8.18 that the result is true in a much broader context. The interested reader can easily write down the results corresponding to Corollary 8.19 for the varieties of associative algebras, alternative algebras and Jordan algebras (see Example 8.10(b), (d) and (e)). 


\section{Two-step loop algebras}

In this section we look more closely at 2-step iterated loop algebras and their centroids. We then conclude with a detailed look at two examples that illustrate several of the concepts studied in this article.

Throughout this section, we assume that $m_{1}$ and $m_{2}$ are positive integers and that $k$ contains a primitive $m_{i}$-th root of unity $\zeta_{m_{i}}, i=1,2$. We use the notation of Section 5 (for iterated loop algebras).

We start with some further notation. Let $k^{\times}=\{\rho \in k \mid \rho \neq 0\}$ be the group of units of $k$. If $\rho \in k^{\times}$, we let

$$
k\left[u_{1}, u_{2}^{ \pm 1}, w\right]_{\rho}
$$

denote the unital associative commutative $k$-algebra presented by the generators $u_{1}, u_{2}, u_{2}^{-1}, w$ subject to the relations

$$
u_{2} u_{2}^{-1}=1 \quad \text { and } \quad w^{2}=\left(u_{1}^{2}-4 \rho\right) u_{2} .
$$

It is clear that the set

$$
\left\{u_{1}^{i_{1}} u_{2}^{i_{1}} w^{j}: i_{1} \in \mathbb{Z}_{\geq 0}, i_{2} \in \mathbb{Z}, j=0,1\right\}
$$

is a $k$-basis for $k\left[u_{1}, u_{2}^{ \pm 1}, w\right]_{\rho}$. It is also easy to verify that the group of units of $k\left[u_{1}, u_{2}^{ \pm 1}, w\right]_{\rho}$ is given by

$$
U\left(k\left[u_{1}, u_{2}^{ \pm 1}, w\right]_{\rho}\right)=\left\{\alpha u_{2}^{i_{2}} \mid \alpha \in k^{\times}, i_{2} \in \mathbb{Z}\right\} .
$$

Indeed, one way to see this is to make use of the multiplicative norm function $N: k\left[u_{1}, u_{2}^{ \pm 1}, w\right]_{\rho} \rightarrow k\left[u_{1}, u_{2}^{ \pm 1}\right]$ defined by $N\left(a_{1}+a_{2} w\right)=a_{1}^{2}-a_{2}^{2} w^{2}$ for $a_{1}, a_{2} \in$ $k\left[u_{1}, u_{2}^{ \pm 1}\right]$, and use the fact that if $u$ is a unit in $k\left[u_{1}, u_{2}^{ \pm 1}, w\right]_{\rho}$ then $N(u)$ is a unit in $k\left[u_{1}, u_{2}^{ \pm 1}\right]$. We leave the details of this to the reader.

The algebra $k\left[u_{1}, u_{2}^{ \pm 1}, w\right]_{\rho}$ is important in the study of iterated loop algebras because of the following fact.

Lemma 9.1. Let $\mathscr{L}=L\left(k, \Sigma_{1}, \Sigma_{2}\right)$ be a 2-step iterated loop algebra based on the algebra $k$, where $\Sigma_{i}$ has modulus $m_{i}$ for $i=1,2$. Then exactly one of the following holds:

(a) $\mathscr{L} \simeq_{k} k\left[t_{1}^{ \pm 1}, t_{2}^{ \pm 2}\right]$ (the algebra of Laurent polynomials in 2 variables).

(b) $\mathscr{L} \simeq_{k} k\left[u_{1}, u_{2}^{ \pm 1}, w\right]_{\rho}$ for some $\rho \in k^{\times}$.

Moreover (a) holds if and only if $z_{1}^{m_{1}} z_{2}^{j} \in \mathscr{L}$ for some $j \in \mathbb{Z}$.

Proof. Note that the group of units in $k\left[t_{1}^{ \pm 1}, t_{2}^{ \pm 1}\right]$ spans the algebra $k\left[t_{1}^{ \pm 1}, t_{2}^{ \pm 1}\right]$, whereas this is not true for the algebra $k\left[u_{1}, u_{2}^{ \pm 1}, w\right]_{\rho}$ (by (9-1)). Thus (a) and (b) cannot hold simultaneously. So it remains to show that either (a) or (b) holds (the final statement will be proved along the way). 
Now as noted in Remark 5.2(iii), we have

$$
\mathscr{L}=L\left(k, \sigma_{1}, \sigma_{2}\right),
$$

where $\sigma_{1}$ is an automorphism of period $m_{1}$ of $\mathscr{L}_{0}=k$, and $\sigma_{2}$ is an automorphism of period $m_{2}$ of $\mathscr{L}_{1}=L\left(k, \sigma_{1}\right)$. Then, since $\sigma_{1}$ is an algebra homomorphism, $\sigma_{1}=1$ and so

$$
\mathscr{L}_{1}=k\left[y_{1}^{ \pm 1}\right], \text { where } y_{1}=z_{1}^{m_{1}} .
$$

Thus $\sigma_{2}$ is an automorphism of period $m_{2}$ of $k\left[y_{1}^{ \pm 1}\right]$. Hence either $\sigma_{2}\left(y_{1}\right)=\rho y_{1}$ for some $m_{2}$-th root of unity $\rho$ in $k^{\times}$or $\sigma_{2}\left(y_{1}\right)=\rho y_{1}^{-1}$ for some $\rho \in k^{\times}$. Moreover (for the proof of the final statement in the proposition) the first of these possibilities holds if and only if $y_{1}$ is homogeneous in the grading $\Sigma_{2}$ determined by $\sigma_{2}$ which in turn holds if and only if $y_{1} z_{2}^{j} \in \mathscr{L}$ for some $j \in \mathbb{Z}$.

Case (a): Suppose that $\sigma_{2}\left(y_{1}\right)=\rho y_{1}$ for some $m_{2}$-th root of unity $\rho$ in $k^{\times}$. Let $n_{2}$ be the order of $\rho$ in $k^{\times}$. Then $n_{2}$ is a divisor of $m_{2}$,

$$
\rho=\zeta_{m_{2}}^{p_{2} r}, \text { where } p_{2}=\frac{m_{2}}{n_{2}},
$$

and $r$ is relatively prime to $n_{2}$ (take $r=0$ if $n_{2}=1$ ). Choose an inverse $s$ for $r$ modulo $n_{2}$ (take $s=0$ if $n_{2}=1$ ). Now the grading $\Sigma_{2}$ of $\mathscr{L}_{1}$ is given by $\mathscr{L}_{1}=$ $\bigoplus_{\bar{j} \in \mathbb{Z}_{m_{2}}}\left(\mathscr{L}_{1}\right)_{\bar{j}}$, where $\left(\mathscr{L}_{1}\right)_{\bar{j}}$ is spanned by the elements $y_{1}^{i}$ with $i \in \mathbb{Z}$ and $\sigma_{2}\left(y_{1}^{i}\right)=$ $\zeta_{m_{2}}^{j} y_{1}^{i}$. But $n_{2}$ and $s$ are relatively prime and so any integer can be expressed in the form $a n_{2}+b s$, where $a, b \in \mathbb{Z}$. Also

$$
\sigma_{2}\left(y_{1}^{a n_{2}+b s}\right)=\rho^{a n_{2}+b s} y_{1}^{a n_{2}+b s}=\rho^{b s} y_{1}^{a n_{2}+b s}=\zeta_{m_{2}}^{p_{2} r b s} y_{1}^{a n_{2}+b s}=\zeta_{m_{2}}^{p_{2} b} y_{1}^{a n_{2}+b s}
$$

and so $y_{1}^{a n_{2}+b s} \in\left(\mathscr{L}_{1}\right) \frac{}{p_{2} b}$. Therefore $\mathscr{L}=L\left(\mathscr{L}_{1}, \sigma_{2}\right)$ is spanned by elements of the form

$$
y_{1}^{a n_{2}+b s} z_{2}^{p_{2} b}, \quad a, b \in \mathbb{Z} .
$$

But $y_{1}^{a n_{2}+b s} z_{2}^{p_{2} b}=\left(y_{1}^{n_{2}}\right)^{a}\left(y_{1}^{s} z_{2}^{p_{2}}\right)^{b}$. Hence we obtain

$$
\mathscr{L}=k\left[t_{1}^{ \pm 1}, t_{2}^{ \pm 2}\right] \text {, where } t_{1}=y_{1}^{n_{2}} \text { and } t_{2}=y_{1}^{s} z_{2}^{p_{2}} .
$$

Case $(b)$ : Suppose that $\sigma_{2}\left(y_{1}\right)=\rho y_{1}^{-1}$ for some $\rho \in k^{\times}$. Then $\sigma_{2}$ has order 2 and so $m_{2}$ is even. Let $p_{2}=\frac{m_{2}}{2}$ and $y_{2}=z_{2}^{p_{2}}$. Then

$$
\mathscr{L}=\left(\mathscr{L}_{1}^{+} \otimes_{k} k\left[\left(y_{2}^{2}\right)^{ \pm 1}\right]\right) \oplus\left(\mathscr{L}_{1}^{-} \otimes_{k} y_{2} k\left[\left(y_{2}^{2}\right)^{ \pm 1}\right]\right),
$$

where $\mathscr{L}_{1}^{ \pm}$is the \pm 1 -eigenspace for $\sigma_{2}$. Now it is clear that $\mathscr{L}_{1}^{+}$has a $k$-basis consisting of the elements $\left(y_{1}+\rho y_{1}^{-1}\right)^{a}, a \geq 0$. Therefore $\mathscr{L}_{1}^{+} \otimes_{k} k\left[\left(y_{2}^{2}\right)^{ \pm 1}\right]$ has basis

$$
\left(y_{1}+\rho y_{1}^{-1}\right)^{a} y_{2}^{2 b}, \quad a, b \in \mathbb{Z}, a \geq 0 .
$$


Also one easily checks that $\mathscr{L}_{1}^{-}=\left(y_{1}-\rho y_{1}^{-1}\right) \mathscr{L}_{1}^{+}$, so $\mathscr{L}_{1}^{-} \otimes_{k} y_{2} k\left[\left(y_{2}^{2}\right)^{ \pm 1}\right]$ has basis

$$
\left(y_{1}-\rho y_{1}^{-1}\right) y_{2}\left(y_{1}+\rho y_{1}^{-1}\right)^{a} y_{2}^{2 b}, \quad a, b \in \mathbb{Z}, a \geq 0 .
$$

Thus, setting

$$
u_{1}=y_{1}+\rho y_{1}^{-1}, u_{2}=y_{2}^{2} \text { and } w=\left(y_{1}-\rho y_{1}^{-1}\right) y_{2},
$$

we see that $\mathscr{L}$ has basis $u_{1}^{a} u_{2}^{b} w^{c}, a \in \mathbb{Z}_{\geq 0}, b \in \mathbb{Z}, c=0,1$. Moreover, one checks directly that $w^{2}=\left(u_{1}^{2}-4 \rho\right) u_{2}$, and so we have identified $\mathscr{L}$ with $k\left[u_{1}, u_{2}^{ \pm 1}, w\right]_{\rho}$.

Remark 9.2. In Case (a) of the proof of Lemma 9.1, the conclusion is an immediate consequence of a more general "erasing theorem" that was proved in [Allison et al. 2004, Theorem 5.1]. We have included the proof above since it is short and selfcontained.

Remark 9.3. If $\rho, \rho^{\prime} \in k^{\times}$, one can show that

$$
k\left[u_{1}, u_{2}^{ \pm 1}, w\right]_{\rho} \simeq_{k} k\left[u_{1}, u_{2}^{ \pm 1}, w\right]_{\rho^{\prime}} \Longleftrightarrow \rho^{\prime} \rho^{-1} \text { is a square in } k^{\times} .
$$

In particular, if $k$ is algebraically closed, the isomorphism class of $k\left[u_{1}, u_{2}^{ \pm 1}, w\right]_{\rho}$ does not depend on $\rho$. In that case Lemma 9.1 tells us that, up to isomorphism, there are exactly two (one step) loop algebras based on $k\left[y_{1}^{ \pm 1}\right]$. This fact is a special case of a more general result about (one step) loop algebras based on the algebra $\mathscr{A}$ of Laurent polynomials $k\left[y_{1}^{ \pm 1}, \ldots, y_{q}^{ \pm 1}\right]$ over an algebraically closed field $k$. Indeed, using the fact that the abstract automorphism group of $\mathscr{A}$ is $\left(k^{\times}\right)^{q} \rtimes \mathrm{GL}_{q}(\mathbb{Z})$ and some techniques from Galois cohomology (see Remark 4.8), one can show that there is an injective map that attaches to each $R$-isomorphism class of loop algebras based on $\mathscr{A}$ an invariant in the set of conjugacy classes of $G L_{q}(\mathbb{Z})$. (When $q=1$, $G L_{q}(\mathbb{Z})$ has exactly two conjugacy classes and one can show that $R$-isomorphism coincides with $k$-isomorphism.) We omit proofs of the statements in this remark, since we will not be using these statements here and since their proofs would take us rather far afield.

Lemma 9.1 together with Theorem 6.2 implies the following more general result:

Proposition 9.4. Let $\mathscr{L}=L\left(\mathscr{A}, \Sigma_{1}, \Sigma_{2}\right)$ be a 2-step iterated loop algebra based on a finite dimensional central simple algebra Al over $k$, where $\Sigma_{i}$ has modulus $m_{i}$ for $i=1,2$. Then exactly one of the following holds:

(a) $C(\mathscr{L}) \simeq_{k} k\left[t_{1}^{ \pm 1}, t_{2}^{ \pm 2}\right]$.

(b) $C(\mathscr{L}) \simeq_{k} k\left[u_{1}, u_{2}^{ \pm 1}, w\right]_{\rho}$ for some $\rho \in k^{\times}$.

Moreover (a) holds if and only if $z_{1}^{m_{1}} z_{2}^{j} \in \bar{C}(\mathscr{L})$ for some $j \in \mathbb{Z}$ (see Remark 6.5).

Definition 9.5. As in Proposition 9.4, let $\mathscr{L}=L\left(\mathscr{A}, \Sigma_{1}, \Sigma_{2}\right)$ be a 2 -step iterated loop algebra based on a finite dimensional central simple algebra $\mathscr{A}$ over $k$, where 
$\Sigma_{i}$ has modulus $m_{i}$ for $i=1,2$. We say that $\mathscr{L}$ is of the first kind (resp. second kind) if $C(\mathscr{L})$ is isomorphic to $k\left[t_{1}^{ \pm 1}, t_{2}^{ \pm 2}\right]$ (resp. $k\left[u_{1}, u_{2}^{ \pm 1}, w\right]_{\rho}$ for some $\rho \in k^{\times}$).

Remark 9.6. (a) It follows from Corollary 6.6 that any 2-step multiloop algebra based on a finite dimensional central simple algebra is of the first kind.

(b) Suppose $k$ is algebraically closed of characteristic 0 and $\mathscr{L}=L\left(\mathscr{A}, \sigma_{1}, \sigma_{2}\right)$ is a 2-step iterated loop algebra based on a finite dimensional central simple Lie algebra $\mathscr{A}$ over $k$, where $\sigma_{i}$ has period $m_{i}$ for $i=1,2$. Then $L\left(\mathscr{A}, \sigma_{1}\right)$ is the derived algebra modulo its centre of an affine Kac-Moody Lie algebra $\mathfrak{g}$ [Kac 1990, Theorem 8.5]. Moreover one can show that the 2-step loop algebra $\mathscr{L}$ is of the first kind in the sense of Definition 9.5 if and only if the automorphism $\sigma_{2}$ of $L\left(\mathscr{A}, \sigma_{1}\right)$ is induced by an automorphism of the first kind of $\mathfrak{g}$ (as defined for example in [Levstein 1988, Part III.1]). Indeed this example is the reason for our choice of terminology.

We conclude by looking at two examples of 2-step iterated loop algebras. These examples illustrate the above proposition (Proposition 9.4) as well as a number of the concepts studied in this article.

Example 9.7. Suppose that $k$ is of characteristic 0. In this example we consider a 2-step iterated loop algebra $\mathscr{L}=L\left(\mathscr{A}, \sigma_{1}, \sigma_{2}\right)$ based on the Lie algebra $\mathscr{A}=\mathrm{sl}_{\ell+1}(k)$ over $k$, where $\ell \geq 1$ and $\sigma_{1}$ and $\sigma_{2}$ have order $m_{1}=m_{2}=2$.

Before beginning it will be convenient to define four commuting automorphisms $\eta_{1}, \eta_{2}, \kappa_{1}$ and $\kappa_{2}$ of $S^{\otimes 2}$ by

$$
\begin{gathered}
\eta_{1}\left(z_{1}^{i_{1}} z_{2}^{i_{2}}\right)=(-1)^{i_{1}} z_{1}^{i_{1}} z_{2}^{i_{2}}, \quad \eta_{2}\left(z_{1}^{i_{1}} z_{2}^{i_{2}}\right)=(-1)^{i_{2}} z_{1}^{i_{1}} z_{2}^{i_{2}}, \\
\kappa_{1}\left(z_{1}^{i_{1}} z_{2}^{i_{2}}\right)=z_{1}^{-i_{1}} z_{2}^{i_{2}} \quad \text { and } \quad \kappa_{2}\left(z_{1}^{i_{1}} z_{2}^{i_{2}}\right)=z_{1}^{i_{1}} z_{2}^{-i_{2}}
\end{gathered}
$$

for $i_{1}, i_{2} \in \mathbb{Z}$. Each of these automorphisms restricts to an automorphism of $k\left[z_{1}^{ \pm 1}\right]$ which we also denote by $\eta_{1}, \eta_{2}, \kappa_{1}$ and $\kappa_{2}$ respectively.

To construct $\mathscr{L}$ we first let $\mathscr{L}_{0}=\mathscr{A}$. Next let $\sigma_{1} \in \operatorname{Aut}(\mathscr{A})$ be defined by $\sigma_{1}(a)=$ $-J a^{t} J$, where

$$
J=\left[\begin{array}{ccc}
0 & \ldots & 1 \\
\vdots & . & \vdots \\
1 & \ldots & 0
\end{array}\right] .
$$

Then $\sigma_{1}$ has order 2 and we set

$$
\mathscr{L}_{1}:=L\left(\mathscr{A}, \sigma_{1}, z_{1}\right)
$$

using the notation of Remark 4.4. Thus $\mathscr{L}_{1}$ is the algebra of fixed points in $\mathscr{A}_{k}$ $k\left[z_{1}^{ \pm 1}\right]$ of the automorphism $\sigma_{1} \otimes \eta_{1}$. (If $k$ is an algebraically closed field of characteristic 0 and $\ell \geq 2$, then $\mathscr{L}_{1}$ is the derived algebra modulo its centre of the affine Kac-Moody Lie algebra of type $A_{\ell}^{(2)}$ [Kac 1990, Chapter 8].) 
Next the automorphisms $1_{\mathscr{A}} \otimes \kappa_{1}$ and $\sigma_{1} \otimes \eta_{1}$ of $\mathscr{A} \otimes k k\left[z_{1}^{ \pm 1}\right]$ commute, so $1_{\mathscr{A}} \otimes \kappa_{1}$ stabilizes $\mathscr{L}_{1}$. We set $\sigma_{2}=1_{\mathscr{A}} \otimes \kappa_{1} \mid \mathscr{L}_{1} \in \operatorname{Aut}_{k}\left(\mathscr{L}_{1}\right)$. Then $\sigma_{2}$ has order 2 , and we set

$$
\mathscr{L}=\mathscr{L}_{2}:=L\left(\mathscr{L}_{1}, \sigma_{2}, z_{2}\right) .
$$

By construction $\mathscr{L}$ is a 2-step iterated loop algebra based on $\mathscr{A}$.

It is clear from the above descriptions of $\mathscr{L}_{1}$ and $\mathscr{L}_{2}$, that $\mathscr{L}$ is the algebra of common fixed points in $\mathscr{A} \otimes_{k} S^{\otimes 2}$ of the automorphisms $\sigma_{1} \otimes \eta_{1}$ and $1_{\mathscr{A}} \otimes \kappa_{1} \eta_{2}$. From this it follows easily that

$$
\mathscr{L}=\left\{x \in \mathrm{sl}_{\ell+1}(K) \mid x^{*}=-x\right\},
$$

where

$$
K=\left(S^{\otimes 2}\right)^{\kappa_{1} \eta_{2}}
$$

is the algebra of fixed points in $S^{\otimes 2}$ of the automorphism $\kappa_{1} \eta_{2}$, and

$$
x^{*}=-J\left(\eta_{1} x\right)^{t} J
$$

for $x \in M_{n}(K)$. (Here $\eta_{1} x$ denotes the matrix obtained from $x$ by applying $\eta_{1}$ to the entries of $x$.) In more geometric language, $\mathscr{L}$ can be viewed as the Lie algebra of $K$-linear transformations of the free $K$-module $K^{\ell+1}$ that are skew relative to the hermitian form $(u, v) \mapsto\left(\eta_{1} u\right)^{t} J v$.

Now by Remark 6.5, the centroid of $\mathscr{L}$ is isomorphic to the algebra

$$
\bar{C}(\mathscr{L})=\left\{u \in S^{\otimes 2} \mid u \cdot \mathscr{L} \subset \mathscr{L}\right\}
$$

of $S^{\otimes 2}$. This together with (9-2) implies that $\bar{C}(\mathscr{L}) \subset K$. But by $(9-3),(u \cdot x)^{*}=$ $\left(\eta_{1} u\right) \cdot x^{*}$ for $u \in K$ and $x \in \operatorname{sl}_{\ell+1}(K)$. Hence it follows from (9-2) and (9-4) that $\bar{C}(\mathscr{L})=K^{\eta_{1}}$. So we have

$$
\bar{C}(\mathscr{L})=\left(S^{\otimes 2}\right)^{\left\langle\eta_{1}, \kappa_{1} \eta_{2}\right\rangle} .
$$

Note also that, by Theorem $7.1, S^{\otimes 2}$ is a free $\bar{C}(\mathscr{L})$-module of rank 4 and

$$
\mathscr{L} \otimes_{\bar{C}(\mathscr{L})} S^{\otimes 2} \simeq \operatorname{si}_{\ell+1}\left(S^{\otimes 2}\right) .
$$

Moreover, by Corollary $8.17, \mathscr{L}$ is a prime pfgc Lie algebra of type $A_{\ell}$ (see Example 8.14).

Finally, note that $\kappa_{1} \eta_{2}\left(z_{1}^{2} z_{2}^{j}\right)=(-1)^{j} z_{1}^{-2} z_{2}^{j} \neq z_{1}^{2} z_{2}^{j}$ and so $z_{1}^{2} z_{2}^{j} \notin \bar{C}(\mathscr{L})$ for $j \in \mathbb{Z}$. Thus $\mathscr{L}$ is of the second kind. (In fact one can check directly that $\bar{C}(\mathscr{L})$ is isomorphic to $k\left[u_{1}, u_{2}^{ \pm 1}, w\right]_{\rho}$ for $\rho=1$.) So $\bar{C}(\mathscr{L})$ is not isomorphic to the algebra of Laurent polynomials in any number of variables (since $\bar{C}(\mathscr{L})$ is not spanned by its units). Hence, by Corollary 6.6, $\mathscr{L}$ is not isomorphic to a multiloop algebra in any number of steps based on a finite dimensional central simple Lie algebra. 
Example 9.8. Suppose that $\ell \geq 1$ and $k$ is a field which contains a primitive $\ell$-th root of unity $\zeta=\zeta_{\ell}$. In this example we consider a 2-step multiloop loop algebra $\mathscr{L}=M\left(\mathscr{A}, \sigma_{1}, \sigma_{2}\right)$ based on the associative algebra $\mathscr{A}=M_{\ell}(k)$ of $\ell \times \ell$-matrices over $k$, where $\sigma_{1}$ and $\sigma_{2}$ have order $m_{1}=m_{2}=\ell$.

First let

$$
a_{1}=\left[\begin{array}{cccc}
1 & 0 & \ldots & 0 \\
0 & \zeta & \ldots & 0 \\
\vdots & \vdots & \ddots & \vdots \\
0 & 0 & \ldots & \zeta^{\ell-1}
\end{array}\right] \text { and } a_{2}=\left[\begin{array}{cccc}
0 & 1 & \ldots & 0 \\
\vdots & \vdots & \ddots & \vdots \\
0 & 0 & \ldots & 1 \\
1 & 0 & \ldots & 0
\end{array}\right]
$$

in $\mathscr{A}$. Then $a_{2} a_{1}=\zeta a_{1} a_{2}, a_{1}^{\ell}=a_{2}^{\ell}=1$, and it is well known that

$$
\left\{a_{1}^{i_{1}} a_{2}^{i_{2}} \mid 0 \leq i_{1}, i_{2} \leq \ell-1\right\}
$$

is a basis for $\mathscr{A}$. (See for example [Draxl 1983, §11].)

Define $\sigma_{i} \in \operatorname{Aut}_{k}(\mathscr{A})$ by $\sigma_{i}(x)=a_{i} x a_{i}^{-1}$ for $x \in \mathscr{A}, i=1,2$. Then $\sigma_{i}\left(a_{i}\right)=a_{i}$, $\sigma_{1}\left(a_{2}\right)=\zeta^{-1} a_{2}$ and $\sigma_{2}\left(a_{1}\right)=\zeta a_{1}$. Hence $\sigma_{1}$ and $\sigma_{2}$ are commuting automorphisms of $\mathscr{A}$ of order $\ell$. Let

$$
\mathscr{L}=M\left(\mathscr{A}, \sigma_{1}, \sigma_{2}\right)
$$

be the multiloop algebra of $\sigma_{1}, \sigma_{2}$ based on $\mathscr{A}$ (with $m_{1}=m_{2}=\ell$ ). To calculate $\mathscr{L}$ explicitly, note that

$$
\sigma_{1}\left(a_{2}^{-i_{1}} a_{1}^{i_{2}}\right)=\zeta^{i_{1}} a_{2}^{-i_{1}} a_{1}^{i_{2}} \quad \text { and } \quad \sigma_{2}\left(a_{2}^{-i_{1}} a_{1}^{i_{2}}\right)=\zeta^{i_{2}} a_{2}^{-i_{1}} a_{1}^{i_{2}}
$$

for $i_{1}, i_{2} \in \mathbb{Z}$. Thus $\mathscr{A}_{\bar{l}_{1}, \bar{i}_{2}}=k a_{2}^{-i_{1}} a_{1}^{i_{2}}$ for $i_{1}, i_{2} \in \mathbb{Z}$. Consequently

$$
\mathscr{L}=\operatorname{span}_{k}\left\{a_{2}^{-i_{1}} a_{1}^{i_{2}} \otimes z_{1}^{i_{1}} z_{2}^{i_{2}} \mid i_{1}, i_{2} \in \mathbb{Z}\right\}=\operatorname{span}_{k}\left\{x_{1}^{i_{1}} x_{2}^{i_{2}} \mid i_{1}, i_{2} \in \mathbb{Z}\right\},
$$

where

$$
x_{1}=a_{2}^{-1} \otimes z_{1}=\left[\begin{array}{cccc}
0 & \ldots & 0 & z_{1} \\
z_{1} & \ldots & 0 & 0 \\
\vdots & \ddots & \vdots & \vdots \\
0 & \ldots & z_{1} & 0
\end{array}\right] \text { and } x_{2}=a_{1} \otimes z_{2}=\left[\begin{array}{cccc}
z_{2} & 0 & \ldots & 0 \\
0 & \zeta z_{2} & \ldots & 0 \\
\vdots & \vdots & \ddots & \vdots \\
0 & 0 & \ldots & \zeta^{\ell-1} z_{2}
\end{array}\right]
$$

in $\mathscr{L}$. Thus $\mathscr{L}$ is the subalgebra of $M_{\ell}\left(S^{\otimes 2}\right)$ generated as an algebra by the matrices $x_{1}^{ \pm 1}, x_{2}^{ \pm 1}$ which satisfy the relations

$$
x_{i} x_{i}^{-1}=x_{i}^{-1} x_{i}=1 \quad \text { and } \quad x_{2} x_{1}=\zeta x_{1} x_{2} .
$$

It follows that $\mathscr{L} \simeq k_{\mathbf{q}}$, where $k_{\mathbf{q}}$ is the algebra presented by the generators $x_{1}, x_{2}$ subject to the relations (9-5). This algebra $k_{\mathbf{q}}$, which is called the quantum torus determined by the matrix

$$
\mathbf{q}=\left[\begin{array}{cc}
1 & \zeta \\
\zeta^{-1} & 1
\end{array}\right]
$$


has arisen in a number of different contexts; see for example [Magid 1978; McConnell and Pettit 1988; Berman et al. 1996; Gao 2000].

Note that by Corollary 6.6, the centroid (= centre) of $\mathscr{L}$ is isomorphic to $\bar{C}(\mathscr{L})=$ $k\left[t_{1}^{ \pm 1}, t_{2}^{ \pm 1}\right]$, where $t_{1}=z_{1}^{\ell}$ and $t_{2}=z_{2}^{\ell}$. Moreover, by Theorem 6.1, $S^{\otimes 2}$ is a free $\bar{C}(\mathscr{L})$-module of rank $\ell^{2}$ and $\mathscr{L} \otimes_{\bar{C}(\mathscr{L})} S^{\otimes 2} \simeq M_{\ell}\left(S^{\otimes 2}\right)$. Consequently (see Corollary 7.4) $\mathscr{L} \simeq k_{\mathbf{q}}$ is a prime Azumaya algebra of constant rank $\ell^{2}$ that is split by the extension $S^{\otimes 2} / k\left[t_{1}^{ \pm 1}, t_{2}^{ \pm 1}\right]$.

Remark 9.9. The fact that the quantum torus $k_{\mathbf{q}}$ (described in the preceding example) is an Azumaya algebra was seen by a different method some time ago in [Magid 1978, Lemma 4]. This information about the algebra $k_{\mathbf{q}}$ is important because it tells us that $k_{\mathbf{q}}$ defines an element $\left[k_{\mathbf{q}}\right]$ of the Brauer group of the ring $k\left[t_{1}^{ \pm 1}, t_{2}^{ \pm 2}\right]$. In fact $\ell \operatorname{Br}\left(k\left[t_{1}^{ \pm 1}, t_{2}^{ \pm 2}\right]\right)$ is cyclic of order $\ell$ and the element $\left[k_{\mathbf{q}}\right]$ is a generator of this group [Magid 1978, Theorem 6].

Remark 9.10. The authors wish to thank John Faulkner for conversations that led to Example 9.8. This example turns out to be a special case of a more general construction of quantum tori and their nonassociative analogs as multiloop algebras. This topic will be investigated in a article in preparation by the present authors together with John Faulkner.

\section{References}

[Allison et al. 1997a] B. N. Allison, S. Azam, S. Berman, Y. Gao, and A. Pianzola, Extended affine Lie algebras and their root systems, Mem. Amer. Math. Soc. 603, Amer. Math. Soc., Providence, RI, 1997. MR 97i:17015 Zbl 0879.17012

[Allison et al. 1997b] B. N. Allison, S. Berman, Y. Gao, and A. Pianzola, "A characterization of affine Kac-Moody Lie algebras”, Comm. Math. Phys. 185:3 (1997), 671-688. MR 98h:17026 Zbl 0879.17013

[Allison et al. 2002] B. Allison, S. Berman, and A. Pianzola, "Covering algebras, I: Extended affine Lie algebras", J. Algebra 250:2 (2002), 485-516. MR 2003d:17026 Zbl 1002.17010

[Allison et al. 2004] B. Allison, S. Berman, and A. Pianzola, "Covering algebras, II: Isomorphism of loop algebras”, J. Reine Angew. Math. 571 (2004), 39-71. MR 2005e:17042 Zbl 1056.17018

[Allison et al. $\geq 2006$ ] B. Allison, S. Berman, and A. Pianzola, "Covering algebras, III: The nullity 2 case". In preparation.

[Benkart and Zelmanov 1996] G. Benkart and E. Zelmanov, "Lie algebras graded by finite root systems and intersection matrix algebras", Invent. Math. 126:1 (1996), 1-45. MR 97k:17044 Zbl 0871.17024

[Berman and Moody 1992] S. Berman and R. V. Moody, "Lie algebras graded by finite root systems and the intersection matrix algebras of Slodowy", Invent. Math. 108:2 (1992), 323-347. MR 93e:17031 Zbl 0778.17018

[Berman et al. 1996] S. Berman, Y. Gao, and Y. S. Krylyuk, "Quantum tori and the structure of elliptic quasi-simple Lie algebras”, J. Funct. Anal. 135:2 (1996), 339-389. MR 97b:17007 Zbl 0847.17009 
[Bourbaki 1972] N. Bourbaki, Commutative algebra, Hermann, Paris, 1972. Reprinted by Springer, Berlin, 1989. MR 90a:13001

[Bourbaki 1974] N. Bourbaki, Algebra, Part I: Chapters 1-3, Hermann, Paris, 1974. Reprinted by Springer, Berlin, 1989. MR 50 \#6689

[Draxl 1983] P. K. Draxl, Skew fields, London Mathematical Society Lecture Note Series 81, Cambridge University Press, Cambridge, 1983. MR 85a:16022 Zbl 0498.16015

[Erickson et al. 1975] T. S. Erickson, W. S. Martindale, and J. M. Osborn, "Prime nonassociative algebras”, Pacific J. Math. 60:1 (1975), 49-63. MR 52 \#3264 Zbl 0355.17005

[Gao 2000] Y. Gao, "Representations of extended affine Lie algebras coordinatized by certain quantum tori”, Compositio Math. 123:1 (2000), 1-25. MR 2002c:17036 Zbl 0958.17008

[Helgason 1978] S. Helgason, Differential geometry, Lie groups, and symmetric spaces, Pure and Applied Mathematics 80, Academic Press, New York, 1978. 2nd ed., Amer. Math. Soc., Providence (RI), 2001. MR 2002b:53081 Zbl 0451.53038

[Jacobson 1962] N. Jacobson, Lie algebras, Interscience, New York, 1962. Reprinted by Dover, New York, 1979. MR 80k:17001 Zbl 0121.27504

[Jacobson 1968] N. Jacobson, Structure and representations of Jordan algebras, Colloquium Publications 39, American Mathematical Society, Providence, R.I., 1968. MR 40 \#4330 Zbl 0218.17010

[Kac 1969] V. G. Kac, “Automorphisms of finite order of semisimple Lie algebras”, Funkcional. Anal. i Priložen. 3:3 (1969), 94-96. In Russian; translated in Funct. Anal. Appl. 3 (1969), 252-254. MR 40 \#4322 Zbl 0274.17002

[Kac 1990] V. G. Kac, Infinite-dimensional Lie algebras, 3rd ed., Cambridge University Press, Cambridge, 1990. MR 92k:17038 Zbl 0716.17022

[Knus 1991] M.-A. Knus, Quadratic and Hermitian forms over rings, Grundlehren der Math. Wissenschaften 294, Springer, Berlin, 1991. MR 92i:11039 Zbl 0756.11008

[Knus and Ojanguren 1974] M.-A. Knus and M. Ojanguren, Théorie de la descente et algèbres d'Azumaya, Lecture Notes in Math. 389, Springer, Berlin, 1974. MR 54 \#5209 Zbl 0284.13002

[Kunz 1985] E. Kunz, Introduction to commutative algebra and algebraic geometry, Birkhäuser, Boston, 1985. MR 86e:14001 Zbl 0563.13001

[van de Leur 2001] J. van de Leur, "Twisted toroidal Lie algebras", preprint, 2001. math/0106119

[Levstein 1988] F. Levstein, "A classification of involutive automorphisms of an affine Kac-Moody Lie algebra”, J. Algebra 114:2 (1988), 489-518. MR 90g:17025 Zbl 0654.17012

[Magid 1978] A. R. Magid, "Brauer groups of linear algebraic groups with characters", Proc. Amer. Math. Soc. 71:2 (1978), 164-168. MR 58 \#5619 Zbl 0393.20029

[McConnell and Pettit 1988] J. C. McConnell and J. J. Pettit, "Crossed products and multiplicative analogues of Weyl algebras", J. London Math. Soc. (2) 38:1 (1988), 47-55. MR 90c:16011 Zbl 0652.16007

[McCrimmon and Zel'manov 1988] K. McCrimmon and E. Zel'manov, “The structure of strongly prime quadratic Jordan algebras", Advances in Mathematics 69:2 (1988), 133-222. MR 89k:17052 Zbl 0656.17015

[Pianzola 2002] A. Pianzola, "Affine Kac-Moody Lie algebras as torsors over the punctured line", Indag. Math. (N.S.) 13:2 (2002), 249-257. MR 2004m:17035 Zbl 1029.17021

[Polikarpov and Shestakov 1990] S. V. Polikarpov and I. P. Shestakov, "Nonassociative affine algebras", Algebra and Logic 29:6 (1990), 458-466. MR 93b:17009 Zbl 0786.17002

[Pollmann 1994] U. Pollmann, "Realisation der biaffinen Wurzelsysteme von Saito in Lie-Algebren", Hamburger Beiträge zur Mathematik (preprint) 29, Universität Hamburg, 1994. 
[Saito and Yoshii 2000] K. Saito and D. Yoshii, "Extended affine root system, IV: Simply-laced elliptic Lie algebras", Publ. Res. Inst. Math. Sci. (Kyoto) 36:3 (2000), 385-421. MR 2002c:17038 Zbl 0987.17012

[Wakimoto 1985] M. Wakimoto, "Extended affine Lie algebras and a certain series of Hermitian representations", preprint, 1985.

[Zhevlakov et al. 1982] K. A. Zhevlakov, A. M. Slin'ko, I. P. Shestakov, and A. I. Shirshov, Rings that are nearly associative, Pure and Applied Mathematics 104, Academic Press, New York, 1982. MR 83i:17001 Zbl 0487.17001

Received December 16, 2004.

BRUCE ALLISON

Department of Mathematical and Statistical Sciences

UNIVERSITY OF ALBERTA

EDMONTON, AB

CANADA T6G 2G1

ballison@math.ualberta.ca

STEPHEN BERMAN

DEPARTMENT OF MATHEMATICS AND STATISTICS

UNIVERSITY OF SASKATCHEWAN

SASKATOON, SK

CANADA S7N 5E6

berman@math.usask.ca

ARTURo PiAnZOLA

DePartment of MATHEMATiCAl and Statistical Sciences

UNIVERSITY OF ALBERTA

EDMONTON, AB

CANADA T6G 2G1

a.pianzola@ualberta.ca 